\title{
Vascular stiffness mechanoactivates YAP/TAZ-dependent glutaminolysis to drive pulmonary hypertension
}

\author{
Thomas Bertero, ${ }^{1}$ William M. Oldham, ${ }^{2}$ Katherine A. Cottrill, ${ }^{3}$ Sabrina Pisano, ${ }^{1}$ Rebecca R. Vanderpool, ${ }^{3}$ Qiujun Yu, ${ }^{3}$ Jingsi Zhao, ${ }^{3}$ \\ Yiyin Tai, ${ }^{3}$ Ying Tang, ${ }^{3}$ Ying-Yi Zhang, ${ }^{4}$ Sofiya Rehman, ${ }^{3}$ Masataka Sugahara, ${ }^{3}$ Zhi Qi, ${ }^{3}$ John Gorcsan III, ${ }^{3}$ Sara O. Vargas, ${ }^{5}$ \\ Rajan Saggar, ${ }^{6}$ Rajeev Saggar, ${ }^{7}$ W. Dean Wallace, ${ }^{6}$ David J. Ross, ${ }^{6}$ Kathleen J. Haley, ${ }^{2}$ Aaron B. Waxman, ${ }^{2}$ Victoria N. Parikh, ${ }^{8}$ \\ Teresa De Marco, ${ }^{9}$ Priscilla Y. Hsue, ${ }^{9}$ Alison Morris, ${ }^{10}$ Marc A. Simon, ${ }^{3}$ Karen A. Norris, ${ }^{11}$ Cedric Gaggioli, ${ }^{1}$ Joseph Loscalzo, ${ }^{4,12}$ \\ Joshua Fessel, ${ }^{13}$ and Stephen Y. Chan ${ }^{3}$
}

'CNRS, UMR7284, INSERM, U1081, Institute for Research on Cancer and Aging, Nice (IRCAN), University of Nice Sophia Antipolis, Medical School, Nice, France. Division of Pulmonary and Critical Care Medicine, Department of Medicine, Brigham and Women's Hospital, Harvard Medical School, Boston, Massachusetts, USA. ${ }^{3}$ Center for Pulmonary Vascular Biology and Medicine, Pittsburgh Heart, Lung, Blood, and Vascular Medicine Institute, Division of Cardiology, Department of Medicine, University of Pittsburgh School of Medicine and University of Pittsburgh Medical Center, Pittsburgh, Pennsylvania, USA. 'ivivision of Cardiovascular Medicine, Department of Medicine, Brigham and Women's Hospital, Harvard Medical School, Boston, Massachusetts, USA. ${ }^{5}$ Department of Pathology, Boston Children's Hospital, Boston, Massachusetts, USA. Departments of Medicine and Pathology, David Geffen School of Medicine, UCLA, Los Angeles, California, USA. 'Department of Medicine, University of Arizona, Phoenix, Arizona, USA. ${ }^{8}$ Division of Cardiology, Department of Medicine, Stanford University, Palo Alto, California, USA. ${ }^{9}$ Division of Cardiology, Department of Medicine, UCSF, San Francisco, California, USA. ${ }^{10}$ Division of Pulmonary and Critical Care Medicine, Department of Medicine, University of Pittsburgh Medical Center, Pittsburgh, Pennsylvania, USA. "Department of Immunology, University of Pittsburgh, Pittsburgh, Pennsylvania, USA. ${ }^{2}$ Laboratory of Systems Pharmacology, Harvard Medical School, Boston, Massachusetts, USA. ${ }^{3}$ Division of Pulmonary and Critical Care Medicine, Department of Medicine, Vanderbilt University Medical Center, Nashville, Tennessee, USA.

Dysregulation of vascular stiffness and cellular metabolism occurs early in pulmonary hypertension (PH). However, the mechanisms by which biophysical properties of the vascular extracellular matrix (ECM) relate to metabolic processes important in PH remain undefined. In this work, we examined cultured pulmonary vascular cells and various types of PHdiseased lung tissue and determined that ECM stiffening resulted in mechanoactivation of the transcriptional coactivators YAP and TAZ (WWTR1). YAP/TAZ activation modulated metabolic enzymes, including glutaminase (GLS1), to coordinate glutaminolysis and glycolysis. Clutaminolysis, an anaplerotic pathway, replenished aspartate for anabolic biosynthesis, which was critical for sustaining proliferation and migration within stiff ECM. In vitro, GLS1 inhibition blocked aspartate production and reprogrammed cellular proliferation pathways, while application of aspartate restored proliferation. In the monocrotaline rat model of $\mathrm{PH}$, pharmacologic modulation of pulmonary vascular stiffness and YAP-dependent mechanotransduction altered glutaminolysis, pulmonary vascular proliferation, and manifestations of PH. Additionally, pharmacologic targeting of GLS1 in this model ameliorated disease progression. Notably, evaluation of simian immunodeficiency virus-infected nonhuman primates and HIV-infected subjects revealed a correlation between YAP/TAZ-CLS activation and PH. These results indicate that ECM stiffening sustains vascular cell growth and migration through YAP/TAZ-dependent glutaminolysis and anaplerosis, and thereby link mechanical stimuli to dysregulated vascular metabolism. Furthermore, this study identifies potential metabolic drug targets for therapeutic development in PH.

\section{Introduction}

Pulmonary hypertension (PH) and its particularly severe subtype pulmonary arterial hypertension (PAH) are poorly understood vascular diseases, characterized by pro-proliferative cellular phenotypes and adverse pulmonary vascular remodeling. Alterations of the vascular extracellular matrix (ECM) are increasingly being recognized as molecular drivers of $\mathrm{PH}$. Dysregulated collagen and elastin production has been observed in both end-stage and early disease (1) and in both proximal and distal vessels (2). Pharmacologic targeting of vascular ECM can improve $\mathrm{PH}$ (3), but the processes that link ECM mechanotransduction (i.e., the processes that enable cells to sense and adapt to external mechanical forces)

Conflict of interest: The authors have declared that no conflict of interest exists. Submitted: January 8, 2016; Accepted: July 7, 2016.

Reference information: / Clin Invest. 2016;126(9):3313-3335. doi:10.1172/JCI86387. to the vasculature are just emerging. Two related transcriptional coactivators inherent to the Hippo signaling pathway, Yes-associated protein 1 (YAP) and TAZ (or WWRT1), are mechanoactivated by stiff ECM and function as central regulators of cellular proliferation and survival across multiple organs, thus modulating tissue growth and development (4). Recently, we found that pulmonary vascular stiffness activates $\mathrm{YAP} / \mathrm{TAZ}$ early in $\mathrm{PH}$, thereby inducing the miR-130/301 family to augment further ECM remodeling and cellular proliferation in vivo (1). While these functional connections are of considerable importance, their molecular mechanisms still remain unclear.

Separately, aerobic glycolysis, a chronic shift in energy production from mitochondrial oxidative phosphorylation to glycolysis, has been described as a pathogenic driver of pulmonary arterial endothelial and smooth muscle proliferation and migration in $\mathrm{PH}$ (as reviewed by ref. 5). Prior mechanistic studies in $\mathrm{PH}$ related to this 
metabolic shift have historically relied on hypoxic disease modeling $(6,7)$. Yet, numerous forms of $\mathrm{PH}$ - subtypes linked to idiopathic or secondary conditions such as predisposing genetic mutations, congenital heart disease, scleroderma, and HIV infection, to name a few - are also characterized by profound metabolic dysregulation in the absence of obvious hypoxic injury. Data are only just emerging (8) regarding the molecular regulators of metabolic dysfunction operating independent of outright hypoxic stress in $\mathrm{PH}$.

Via this perspective, increasing evidence suggests a central connection of YAP/TAZ activity with cellular metabolism in contexts beyond $\mathrm{PH}$, including processes related to glucose consumption and aerobic glycolysis $(9,10)$. However, increased glycolysis alone is insufficient to meet the total metabolic demands of such proliferating cells. The tricarboxylic acid (TCA) cycle also serves as a source of energy production and provides a critically important reservoir of substrates for the biosynthesis of amino acids, carbohydrates, and lipids (11). Continued functioning of the TCA cycle requires the replenishment of carbon intermediates. This replenishment, or anaplerosis, is accomplished via 2 major pathways: glutaminolysis (deamidation of glutamine via the enzyme glutaminase [GLS1]) and carboxylation of pyruvate to oxaloacetate via ATP-dependent pyruvate carboxylase (PC). Specifically, glutaminolysis via GLS1 activity contributes to anaplerosis by allowing for mobilization of cellular energy, carbon, and nitrogen, particularly in rapidly proliferating cells (12), and serves as a critical process in transformed cells that have switched their metabolism from oxidative phosphorylation to glycolysis in order to maintain cell growth and viability (13). The particular ability of glutaminolysis (and/or pyruvate carboxylation) to support aspartate production for direct induction of proliferation has recently been reported in malignant cells $(14,15)$. In $\mathrm{PH}$, dysregulation of glutaminolysis in the failing right ventricular cardiomyocyte has been observed (16). Yet, the pathogenic importance of glutamine metabolism, particularly as driven by pulmonary vascular stiffening or in the context of YAP/TAZ activation, has not been defined.

Consequently, in this study we investigated whether a metabolic response to pulmonary vascular stiffness controls cellular proliferation in PH. Specifically, we aimed to determine whether ECM stiffness directly modulates both glycolysis and anaplerosis in order to meet the metabolic demands of the pro-proliferative disease state, thus effectively linking vascular stiffness and metabolic dysfunction as 2 integrally related molecular drivers of this enigmatic disease.

\section{Results}

Mechanical stimuli regulate metabolic reprogramming in pulmonary vascular endothelial and smooth muscle cells. To determine whether mechanical/physical cues conveyed by ECM stiffness modulate vascular cell metabolism, we performed metabolic screening of pulmonary vascular cell types cultured on soft or stiff matrix (Figure 1 and Supplemental Figure 1; supplemental material available online with this article; doi:10.1172/JCI86387DS1). Here, soft matrix was defined by a Young's (elastic) modulus of $1 \mathrm{kPa}$, consistent with our prior studies of the stiffness of nondiseased pulmonary arterioles in rodents (1); stiff matrix was defined as $50 \mathrm{kPa}$. Via extracellular flux analysis, oxygen consumption rate and extracellular acidification rate (a surrogate marker of glycolysis) were assessed in pulmonary arterial endothelial cells (PAECs) (Figure $1, \mathrm{~A}-\mathrm{C}$ ). As reflected by extracellular acidification rate quantification (Figure 1, A and B), ECM stiffness increased basal glycolysis while concomitantly decreasing glycolytic reserve capacity, calculated as the difference between oligomycin A-induced extracellular acidification rate and basal extracellular acidification rate. Thus, cells on stiff matrix displayed a glycolytic flux closer to their maximal rate compared with cells on soft matrix. Alternatively, increased ECM stiffness significantly decreased basal oxygen consumption rate, ATP-dependent oxygen consumption rate (difference between basal oxygen consumption rate and oligomycin A-inhibited oxygen consumption rate), and maximal oxygen consumption rate (reflected by induction via carbonyl cyanide- $p$-trifluoromethoxyphenylhydrazone [FCCP]) (Figure 1, $\mathrm{A}$ and $\mathrm{C}$ ), thus reflecting a decrease in mitochondrial oxidative phosphorylation. Corresponding with these metabolic changes, stiff matrix decreased overall mitochondrial potential (Figure 1D). Similar results were observed for pulmonary arterial smooth muscle cells (PASMCs) (Supplemental Figure 1, A-G). Taken together, stiff conditions act as a mechanical stimulus to increase glycolysis and decrease mitochondrial oxidative phosphorylation.

To determine the activity of glycolysis, anaplerosis, and the TCA cycle under these same mechanical conditions, we measured candidate intracellular amino acids and metabolites (Figure 1E) by liquid chromatography-tandem mass spectrometry (LC-MS/MS) in PAECs. Consistent with increased glycolysis and decreased oxidative phosphorylation in stiff conditions, we observed an increase of lactate/pyruvate ratio (Figure $1 \mathrm{~F}$ ). Further consistent with decreased oxidative phosphorylation (Figure 1A), stiff matrix decreased succinate levels (Figure 1G) and increased lactate production (Figure 1H). Importantly, ECM stiffening also decreased intracellular glutamine accompanied by a robust increase of glutamate and aspartate (Figure 1G), consistent with a putative anaplerotic process accompanying accelerated glycolysis. Levels of $3 \mathrm{key}$ enzymes in PAECs - lactate dehydrogenase A (LDHA), both GLS1 isoforms (KGA and GAC), and PC - implicated in both glycolysis (LDHA) and anaplerosis (GLS1 and PC) were elevated (Figure 1, I-K, and Supplemental Figure 1K) in stiff matrix. Notably, only GLS1, but not GLS2, was responsive to stiff matrix (Supplemental Figure 1L). As above, similar results were obtained for PASMCs (Supplemental Figure 1, H-K). Thus, exposure to stiff matrix not only alters glycolysis and oxidative phosphorylation but also controls anaplerotic replenishment of amino acids.

ECM stiffness depends on YAP/TAZ to control metabolism. Given our prior findings that YAP and TAZ act as mechanosensors in pulmonary vascular cells (1), we investigated whether they are important in modulating the effects of ECM stiffening on metabolic reprogramming. In PAECs in stiff matrix, knockdown of YAP/TAZ (Figure 2A) decreased extracellular lactate and lactate/ pyruvate ratio (Figure 2, B-D, and Supplemental Figure 2), reflecting its control over glycolysis. YAP/TAZ knockdown also blunted the effects of stiff ECM on intracellular glutamine, glutamate, and aspartate (Figure 2C). Mitochondrial membrane potential was also sustained during YAP/TAZ knockdown in stiff matrix (Figure 2E). Conversely, in PAECs grown in soft matrix, stable expression of YAP (pYAP) (Figure 2F) increased extracellular lactate and lactate/pyruvate ratio (Figure 2, G-I); decreased glutamine and 
A

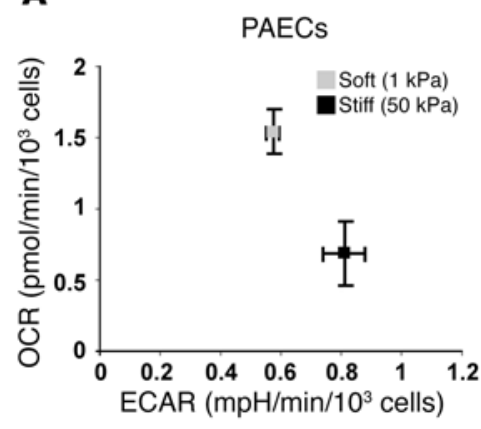

E

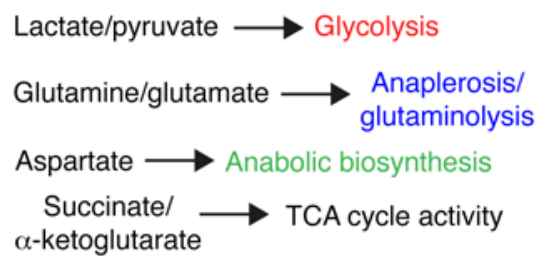

B

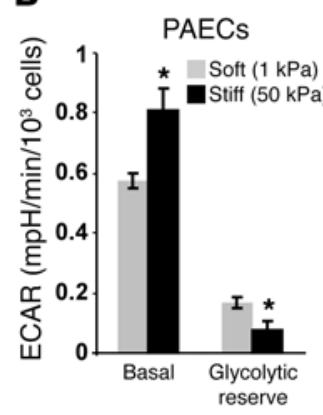

C

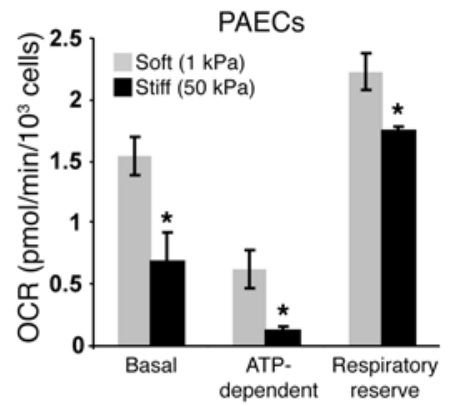

D

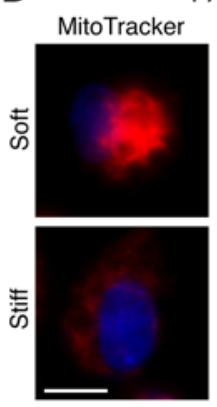

PAECS

Soft $(1 \mathrm{kPa})$
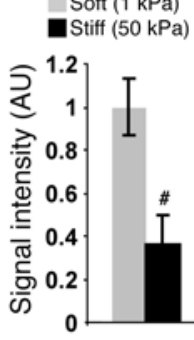
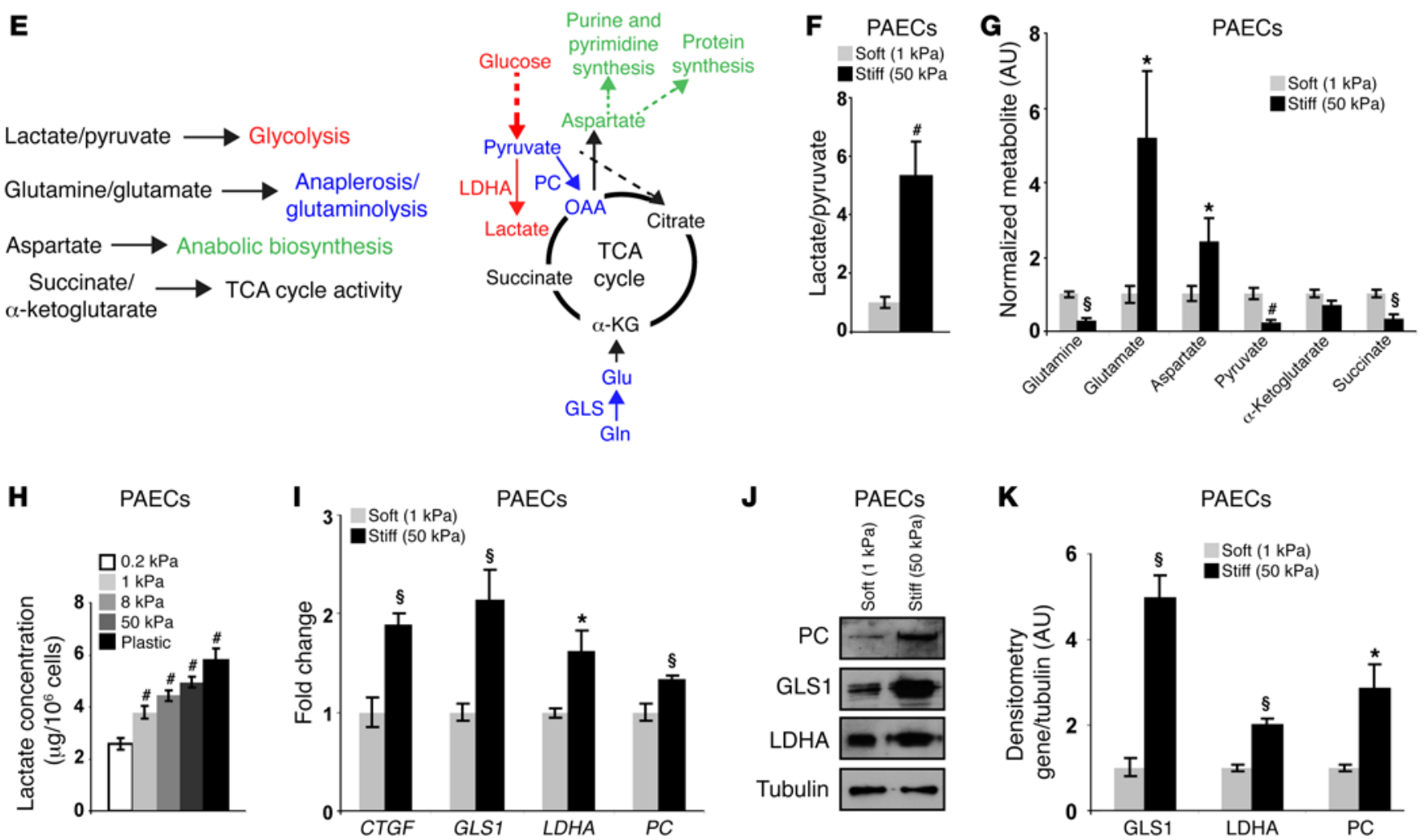

Figure 1. ECM stiffening activates glycolysis and glutaminolysis. (A) By extracellular flux analysis, PAECs cultivated in stiff matrix displayed decreased oxygen consumption rate (OCR) and increased extracellular acidification rate (ECAR), reflective of glycolysis. (B) PAECs cultivated in stiff matrix displayed increased basal glycolysis and corresponding decreased glycolytic reserve (as assessed by the difference between oligomycin A-induced ECAR and basal ECAR). (C) Basal OCR, ATP-dependent OCR (difference between basal OCR and oligomycin A-inhibited OCR), and respiratory reserve (maximal FCCPinduced OCR) were decreased in PAECs in stiff matrix. (D) MitoTracker (Thermo Fisher Scientific) labeling confirmed a decrease of mitochondrial activity in PAECs in stiff matrix. (E) Major metabolites in anaplerosis and glycolysis were measured in this study. GLS and PC generate the major anaplerotic metabolites (blue), feeding into the TCA cycle (black) and supporting the anabolic demand for biosynthesis (green). LDHA modulates glycolysis (red). $\alpha$-KG, $\alpha$-ketoglutarate; OAA, oxaloacetic acid. (F) In PAECs in stiff matrix, increased lactate/pyruvate ratio was observed, consistent with increased glycolysis and decreased oxidative phosphorylation. (C) In these same cells, glutamine, pyruvate, and succinate were decreased, while glutamate and aspartate were increased. (H) Released lactate was progressively increased in PAECs cultivated in an increasing gradient of matrix stiffness. (I-K) In PAECs, GLS1, LDHA, and $P C$ expression was increased by matrix stiffening, as confirmed by RT-quantitative PCR (RT-qPCR) (CTCF, positive control) (I), and by immunoblot and densitometry (J and $\mathbf{K})$. In all panels, mean expression in controls (soft matrix) was assigned a fold change of 1, to which relevant samples were compared. Data are expressed as the mean \pm SEM $\left({ }^{*} P<0.05,{ }^{\circledR} P<0.01,{ }^{\#} P<0.001\right)$ of at least 3 independent experiments performed in triplicate. Paired samples were compared by 2-tailed Student's $t$ test, while 1-way ANOVA and post-hoc Tukey's tests were used for group comparisons. Scale bar: $20 \mu \mathrm{m}$. See also Supplemental Figure 1 (similar results were found in PASMCs).

increased glutamate and aspartate (Figure 2H); and consequently, decreased mitochondrial membrane potential (Figure 2J). Notably, the same pathways of glycolysis and glutaminolysis mechanically controlled by stiff ECM in PAECs were activated by YAP/ TAZ in PASMCs (Supplemental Figure 2).
Several putative binding sites for YAP/TAZ complexes (TEAD sites) were revealed by sequence analysis of the promoter regions of key metabolic enzymes responsible for glycolysis and glutaminolysis - LDHA, GLS1, and PC (Figure 3A). ChIP-quantitative PCR demonstrated the direct binding of YAP on at least 

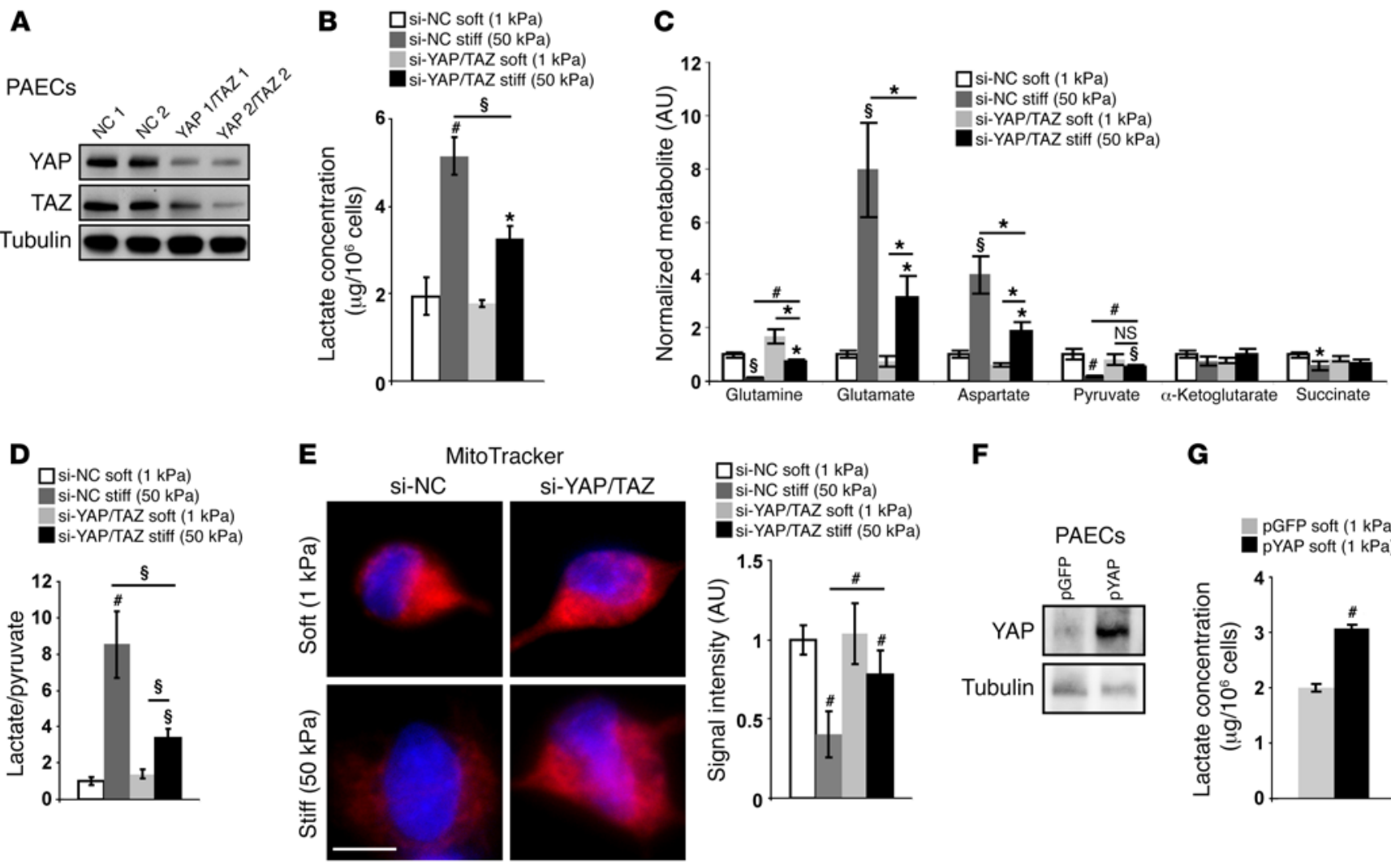

$\mathbf{F}$

G

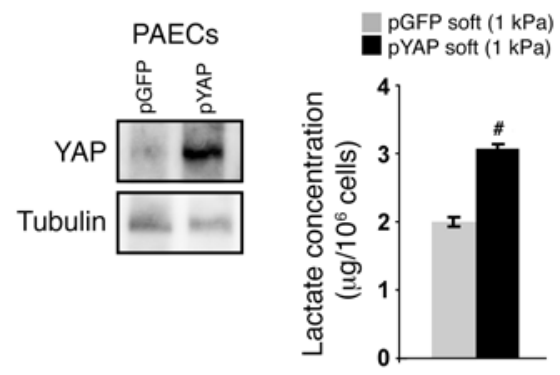

H

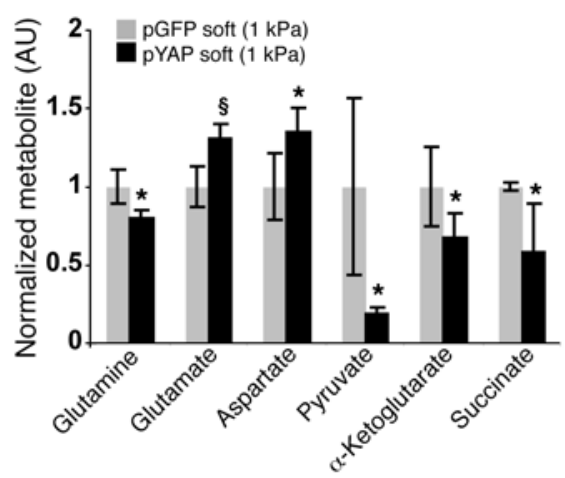

I
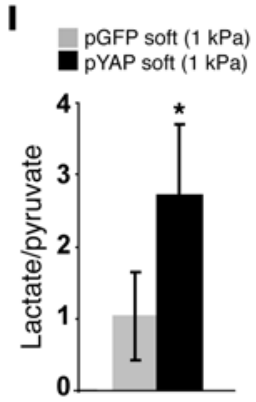

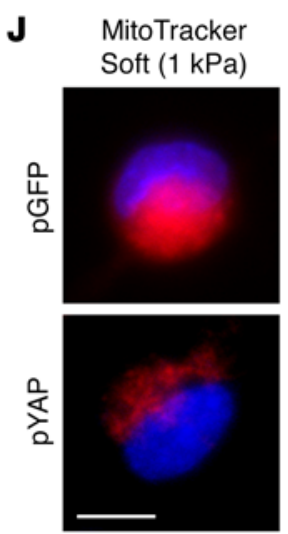

Figure 2. A metabolic switch induced by ECM stiffening is coordinated by the mechanoactivation of YAP/TAZ. (A) Immunoblot analysis confirmed the knockdown of YAP and TAZ by 2 independent siRNA sequences in PAECs. (B-E) PAECs were cultured in soft or stiff matrix. Released lactate was increased in stiff matrix, but such increase was blunted by siRNA knockdown of YAP/TAZ (B). In stiff matrix, YAP/TAZ knockdown also blunted specific metabolite alterations reflective of anaplerotic (C) and glycolytic activity as reflected by the lactate/pyruvate ratio (D). MitoTracker labeling confirmed that YAP/TAZ knockdown reversed the alteration of mitochondrial membrane potential triggered by stiff matrix (E). (F) Immunoblot analysis confirmed the forced expression of YAP in PAECs infected with a lentiviral vector containing the YAP coding sequences (pYAP) compared with cells infected with a control vector (pGFP). (C) In PAECs cultivated on soft matrix, forced expression of YAP (pYAP) increased lactate compared with control vector (pGFP). (H and I) In similarly treated PAECs, YAP decreased glutamine, pyruvate, and succinate, increased glutamate and aspartate (H), and increased lactate/ pyruvate ratio (I). (J) MitoTracker labeling confirmed that YAP (pYAP) in soft matrix altered PAEC mitochondrial membrane potential. In all panels, mean expression in control groups (si-NC, pGFP cultivated on soft matrix) was assigned a fold change of 1, to which relevant samples were compared. Data are expressed as mean \pm SEM $\left({ }^{*} P<0.05,{ }^{\circledR} P<0.01,{ }^{\#} P<0.001\right)$ of at least 3 independent experiments performed in triplicate. Paired samples were compared by 2 -tailed Student's $t$ test, while 1-way ANOVA and post-hoc Tukey's tests were used for group comparisons. Scale bars: $20 \mu \mathrm{m}$. See also Supplemental Figure 2 (similar results were found in PASMCs).

1 site for each gene (Figure 3B). Correspondingly, siRNA knockdown of YAP/TAZ in PAECs (Figure 3, C-E) and in PASMCs (Supplemental Figure 2) decreased target gene expression, while forced YAP expression in PAECs in soft matrix increased their levels (Figure 3, F-H). Notably, only knockdown of YAP and TAZ together, but not YAP or TAZ alone, was sufficient to decrease the target gene expression (Supplemental Figure 2). Taken together, YAP and TAZ are integral to the mechano-triggered, 
A

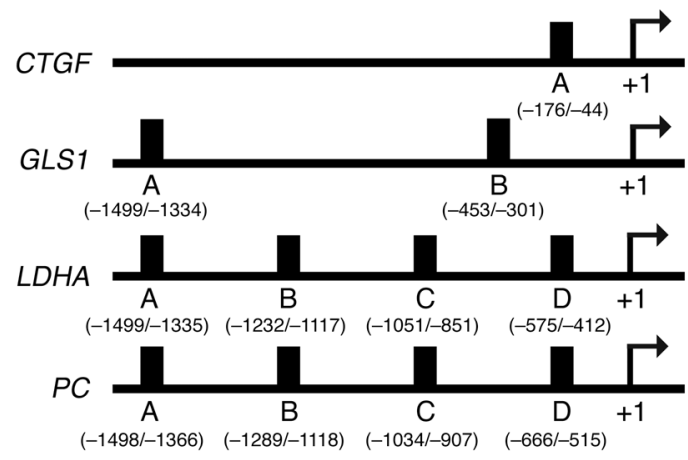

C

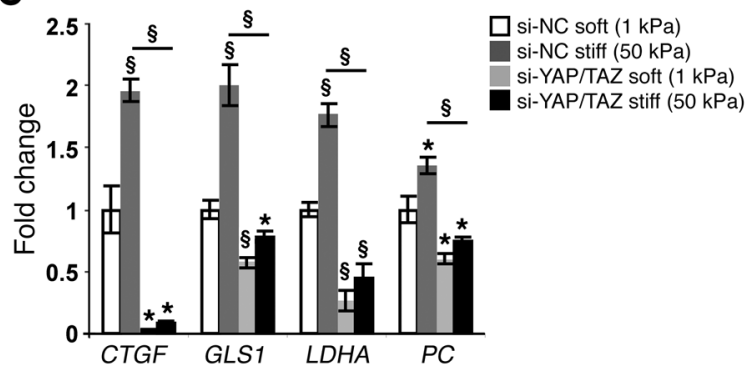

B 3

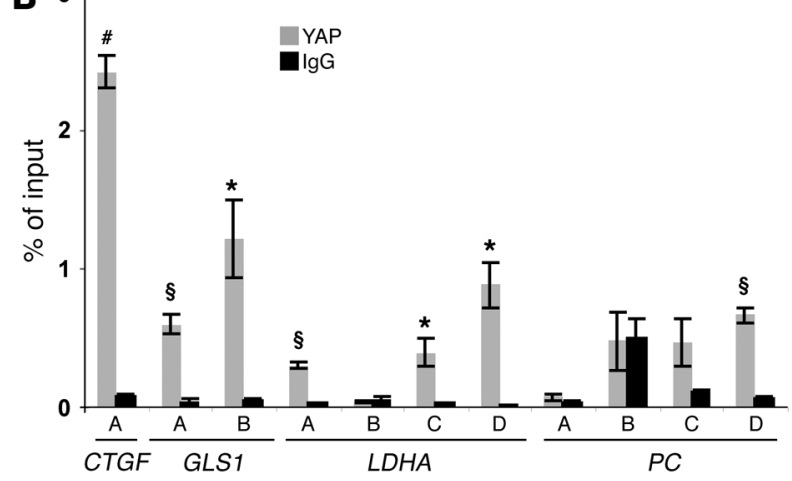

D

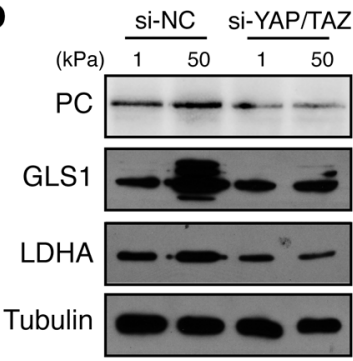

E

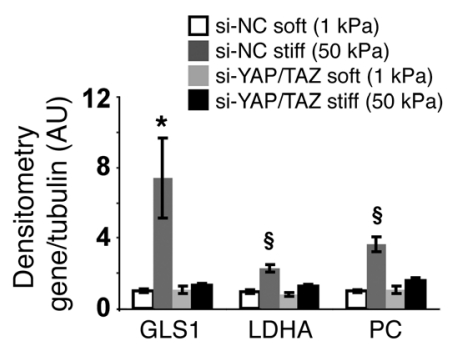

F

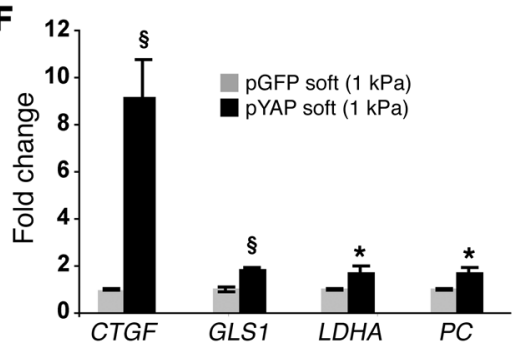

G

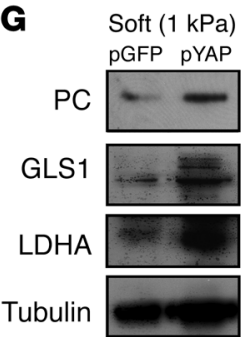

H

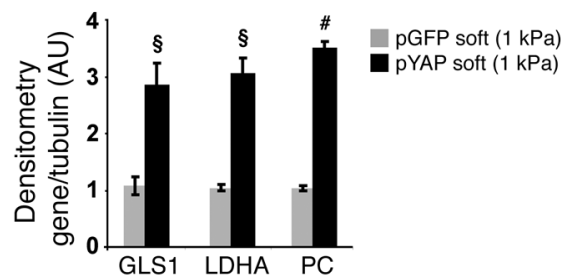

Figure 3. YAP and TAZ control the transcription of key metabolic enzymes. (A) Sequence analysis predicted the presence of TEAD binding sites (labeled as A-D) in the promoter regions of GLS1, LDHA, and PC. (B) ChIP-qPCR confirmed the presence of TEAD/YAP binding sites in the GLS1, LDHA, and PC promoter regions. CTGF, a known YAP target, was used as a positive control. Results are expressed as percentage of total input DNA prior to immunoprecipitation with anti-YAP or anti-IgG control. (C-E) RT-qPCR (C) accompanied by immunoblotting (D) and densitometry (E) revealed that increased GLS1, LDHA and PC expression in PAECs in stiff matrix was blunted by YAP/TAZ knockdown. (F-H) RT-qPCR (F) and immunoblotting and densitometry (G and $\mathbf{H})$ revealed that YAP (pYAP) increased GLS1, LDHA, and PC expression in PAECs in soft matrix. In all panels, mean expression in control groups (si-NC, pGFP cultivated on soft matrix) was assigned a fold change of 1 , to which relevant samples were compared. Data are expressed as the mean \pm SEM $\left({ }^{*} P<0.05\right.$, $\left.\$ P<0.01,{ }^{\#} P<0.001\right)$ of at least 3 independent experiments performed in triplicate. Paired samples were compared by 2-tailed Student's $t$ test, while 1-way ANOVA and post-hoc Tukey's tests were used for group comparisons. See also Supplemental Figure 2 (similar results were found in PASMCs).

glycolytic and glutaminolytic metabolic reprogramming events initiated by ECM stiffness.

Increased GLS1 expression and glutaminolysis are critical for sustaining glycolysis and cell proliferation in a stiff environment. To determine whether GLS1 is critical for stiffness-induced and YAP/ TAZ-dependent glutaminolysis, PAECs were cultivated in soft or stiff matrix and exposed to known pharmacologic inhibitors of both isoforms of GLS1 - BPTES [bis-2-(5-phenylacetamido-1,3,4-thiadiazol-2-yl)ethyl sulphide] (17), DON (6-diazo-5-oxo-L-norleucine) (18), C968 (glutaminase inhibitor, compound 968) (19) (Figure 4, A-C), or siRNA (si-GLS1; Figure 4, D-G). As quantified by LC-MS/MS, inhibition of GLS1 in PAECs blunted the stiffnessinduced processes of glutamine consumption, glutamate production, and aspartate production (Figure 4, A and E). GLS1 inhibition also decreased glycolysis in stiff matrix, as indicated by decreased extracellular lactate and lactate/pyruvate ratio (Figure 4, B, C, F, and G). Similar alterations of metabolic activity were observed when GLS1 was inhibited in PASMCs (Supplemental Figure 3, A-F).

To identify downstream molecular processes regulated by GLS1, expression array analysis and pathway enrichment (20) of PAECs exposed to si-GLS1 on stiff matrix revealed a systematic reprogramming of multiple pathways, notably downregulating cell cycle genes controlling proliferative capacity and factors involved in extracellular matrix organization controlling cell migration (Figure 5A, Supplemental Figure 4, A and B, and Supplemental Table 1). As assessed by cell count (Figure 5, B, C, F, and G), BrdU pulse (Figure 5, D and H), caspase-3/7 activity (Supplemental Figure $4 \mathrm{C}$ ), and proliferating cell nuclear antigen (PCNA)/cleaved caspase-3 double staining (Supplemental Figure 4, D and E), GLS1 inhibition had a negligible effect on apoptosis and on proliferation 
A

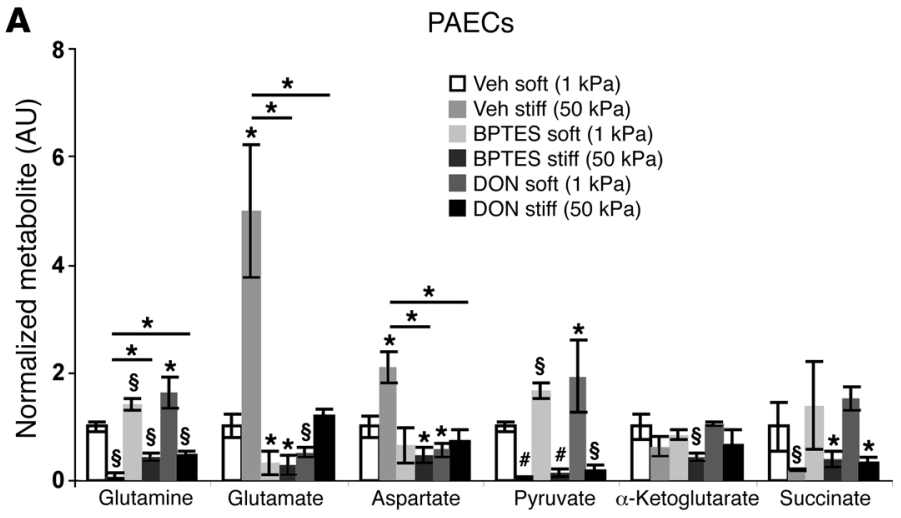

B

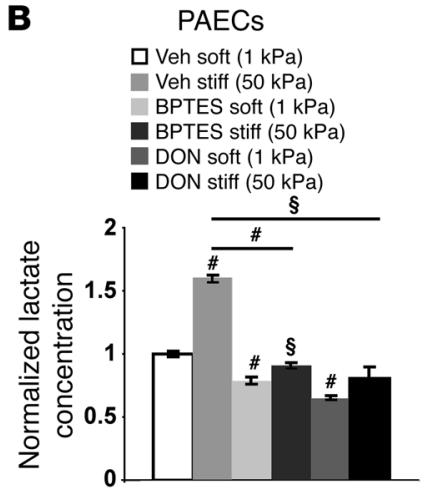

C

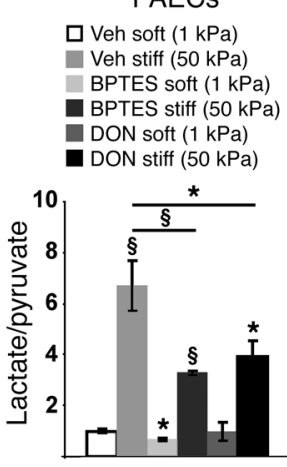

D

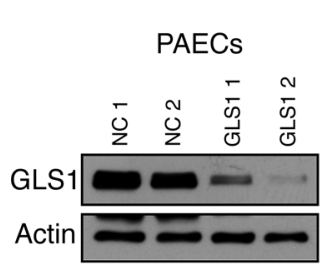

E

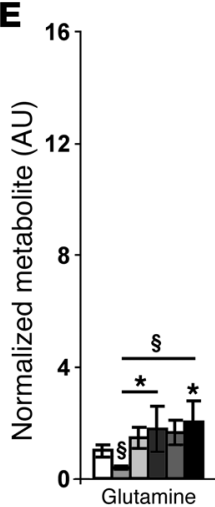

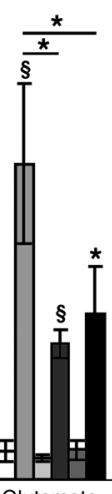

Glutamate

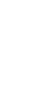

$\square$ si-NC soft $(1 \mathrm{kPa})$ si-NC stiff $(50 \mathrm{kPa})$ si-GLS1 1 soft (1 kPa) - si-GLS1 1 stiff (50 kPa) si-GLS1 2 soft ( $1 \mathrm{kPa})$ - si-GLS1 2 stiff (50 kPa)

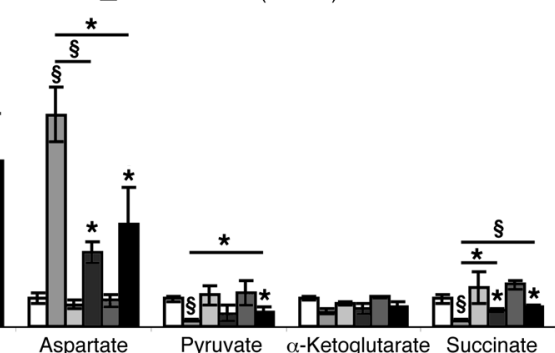

$\mathbf{F}$

\section{G}

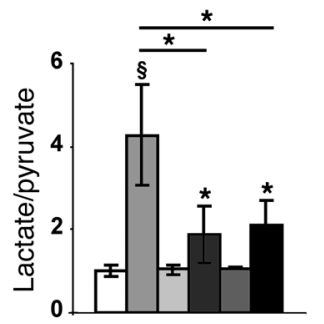

Figure 4. Pharmacologic or genetic inhibition of GLS1 blunts the upregulation of glutaminolysis in stiff matrix. (A-C) In PAECs, targeted LC-MS/MS revealed that pharmacologic inhibition of GLS1 (BPTES or DON) blunted the alterations of metabolite expression in stiff matrix. Specifically, compared with stiff matrix control (si-NC stiff), GLS1 inhibition increased glutamine, pyruvate, and succinate, decreased glutamate and aspartate (A), and decreased extracellular lactate (B) as well as lactate/pyruvate ratio (C). (D) Immunoblot analysis confirmed the knockdown of GLS1 by 2 independent siRNA sequences. (E-C) In PAECs, GLS1 knockdown blunted the alterations of metabolite expression in stiff matrix, increasing glutamine, pyruvate, and succinate; decreasing glutamate and aspartate $(\mathbf{E})$; and decreasing extracellular lactate and lactate/pyruvate ratio (F and $\mathbf{G})$. In all panels, mean expression in control groups (vehicle, si-NC cultivated on soft matrix) was assigned a fold change of 1 , to which relevant samples were compared. Data are expressed as the mean \pm SEM $\left({ }^{*} P<0.05,{ }^{\$} P<0.01,{ }^{*} P<0.001\right)$ of at least 3 independent experiments performed in triplicate. One-way ANOVA and post-hoc Tukey's tests were used for group comparisons.

in soft matrix but blunted proliferation on stiff matrix in PAECs. Moreover, corresponding to the transcriptomic results affecting matrix organization (Figure 5A and Supplemental Figure 4A), GLS1 inhibition, achieved via siRNA (Figure 5E) or pharmacologic means (Figure 5I), inhibited cell migration. Similar effects were observed in PASMCs (Supplemental Figure 3, G and H).

To investigate whether the anaplerotic production of glutamate and aspartate is central to the actions of GLS1 to sustain proliferation, glutamate or aspartate supplementation was performed in cells lacking GLS1 or YAP/TAZ. Consistent with our results (Figure 5) and prior observations (21), siRNA knockdown of GLS1 or YAP/TAZ decreased proliferation in either PAECs or PASMCs, as assessed by cell count (Figure 6, A-C, and Supplemental Figure 5) and quantitation of the proliferation marker PCNA (Figure 6, D and E). Importantly, in cells with diminished GLS1 or YAP/ TAZ (Figure 6, A-E, and Supplemental Figure 5), cellular proliferation was at least partially restored by glutamate and more fully restored by aspartate supplementation. Aspartate supplementation similarly reversed the reduced cell migration of GLS1- or YAP/TAZ-deficient PAECs on stiff matrix (Figure 6F). Collectively, these results demonstrate that GLS1 and its control of glutamate and aspartate production by glutaminolysis are essential for metabolic reprogramming and consequent vascular cell proliferation and migration specific to stiff matrix exposure. 
A

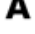

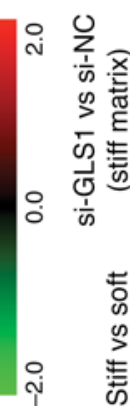

Extracellular matrix organization फेंक

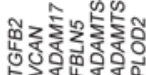

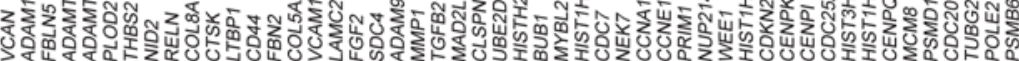

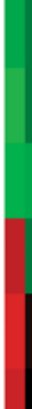

D

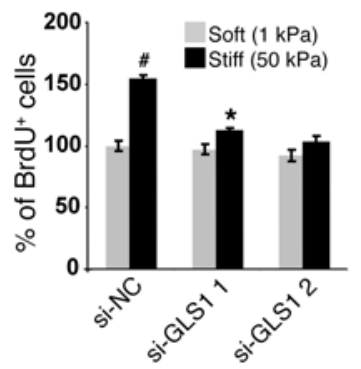

E

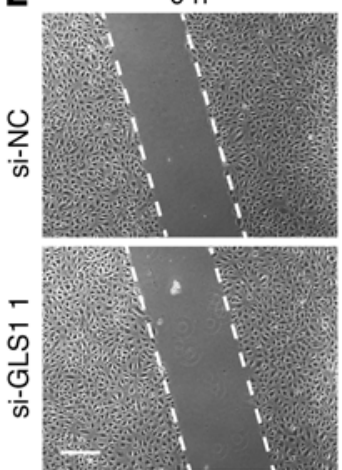

Cell cycle

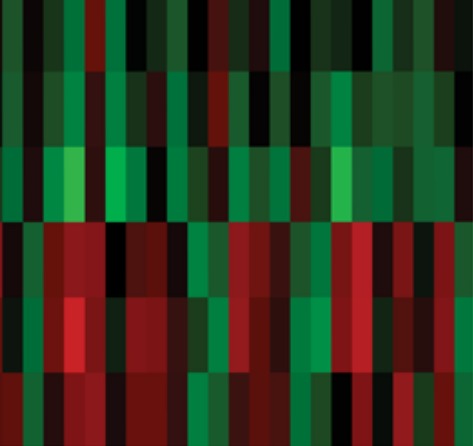

B

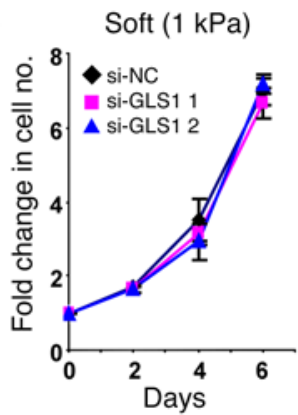

C

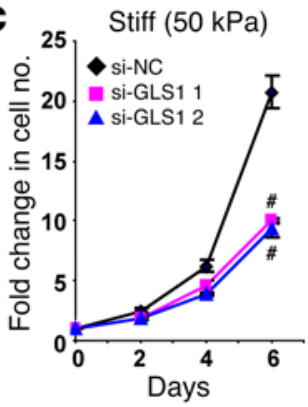

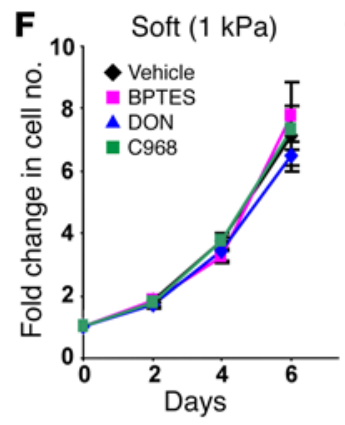

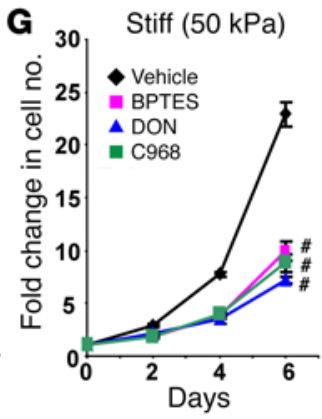

I
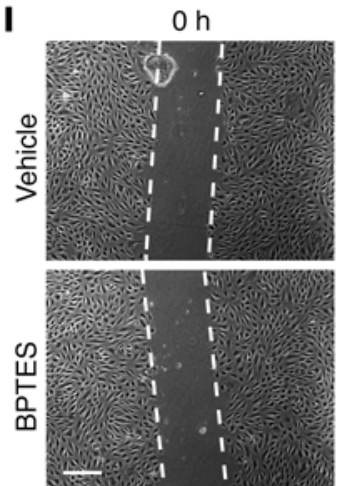

$10 \mathrm{~h}$

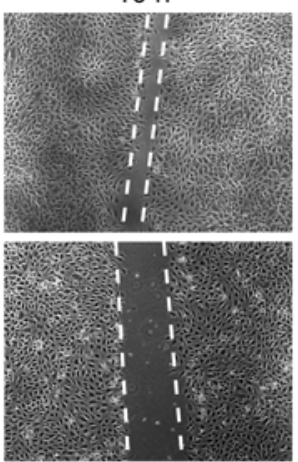

$10 \mathrm{~h}$

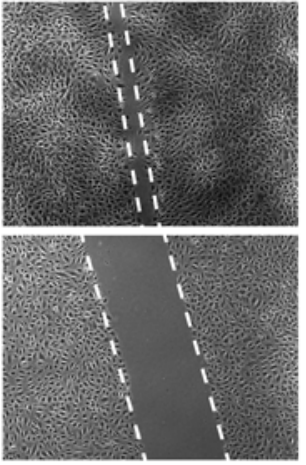

$\square$ si-NC

Si-GLS1 1

- $\mathrm{si}-\mathrm{GLS} 12$

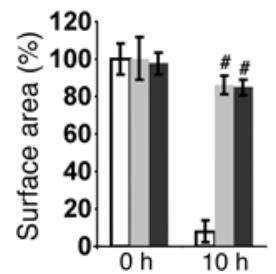

H

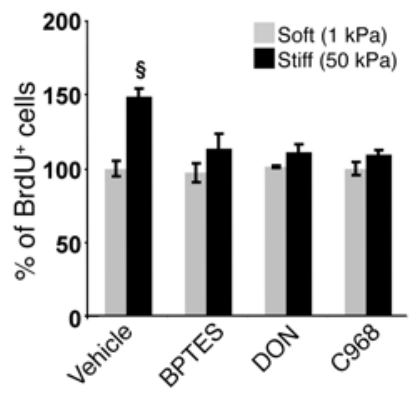

Figure 5. Inhibition of GLS1 decreases glutaminolysis in order to sustain cellular proliferation in stiff matrix. (A) Transcriptomic analysis (heatmap) of PAECs cultivated on soft or stiff matrix ( $n=3$ per group) revealed a programmatic alteration of genes associated with ECM organization and proliferation (cell cycle). Notably, these changes were blunted by GLS1 knockdown on stiff matrix. (B-D) GLS1 knockdown blunted PAEC proliferation on stiff (C), but not soft (B), matrix, as revealed by cell counting (B and $\mathbf{C}$ ) and BrdU pulse experiments (D). (E) As assessed by scratch assay, GLS1 knockdown also inhibited PAEC migration. (F-I) Similarly, pharmacologic inhibition of GLS blunted PAEC proliferation on stiff (G), but not soft (F), matrix (F-H) and inhibited PAEC migration (I). In all panels, mean expression in control groups (si-NC or vehicle cultivated on soft matrix) was assigned a fold change of 1, to which relevant samples were compared. Data are expressed as the mean \pm SEM $\left({ }^{*} P<0.05,{ }^{\S} P<0.01,{ }^{\#} P<0.001\right)$ of at least 3 independent experiments performed in triplicate. Paired samples were compared by 2-tailed Student's $t$ test, while 1-way ANOVA and post-hoc Tukey's tests were used for group comparisons. Scale bars: $200 \mu \mathrm{m}$. See also Supplemental Figures 3 and 4. 

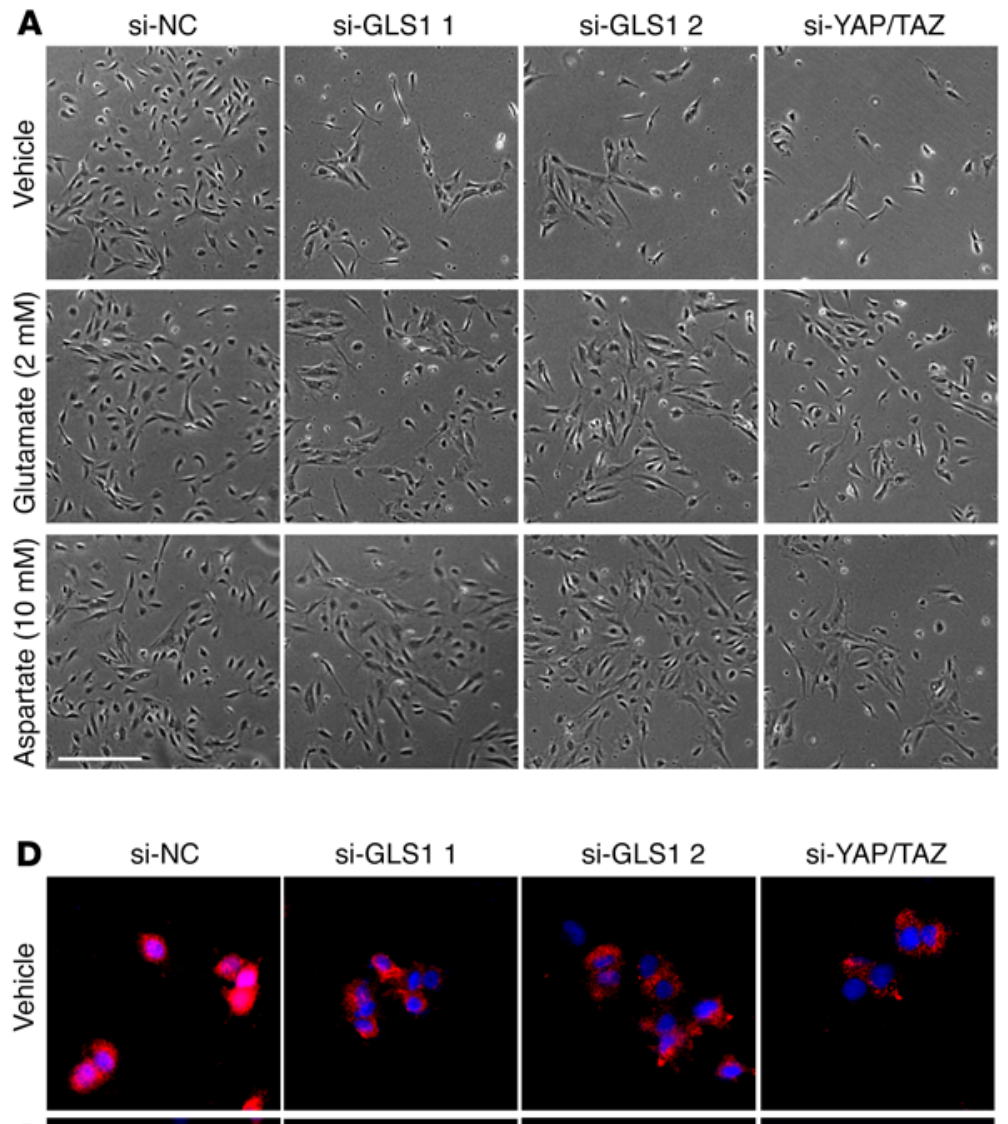
si-GLS1 1
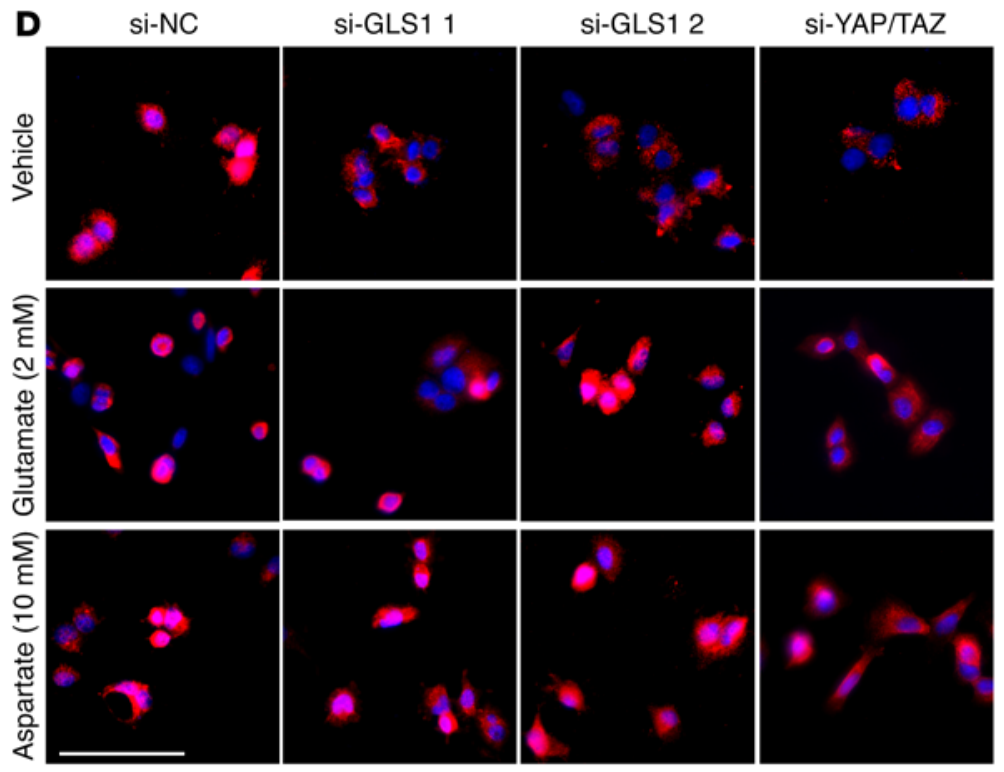

F

si-NC
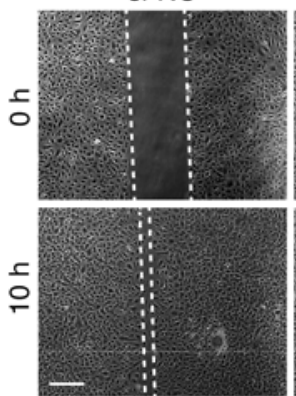

si-GLS1
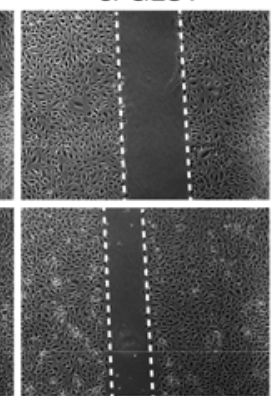

Si-YAP/TAZ

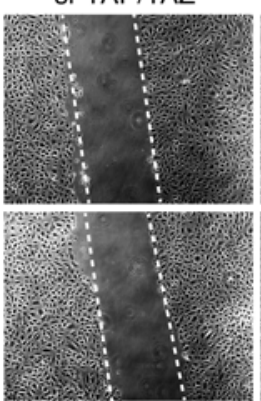

$\frac{+A}{\text { si-GLS1 }}$

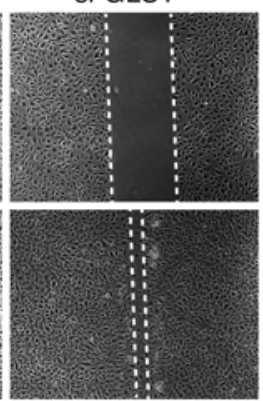

B
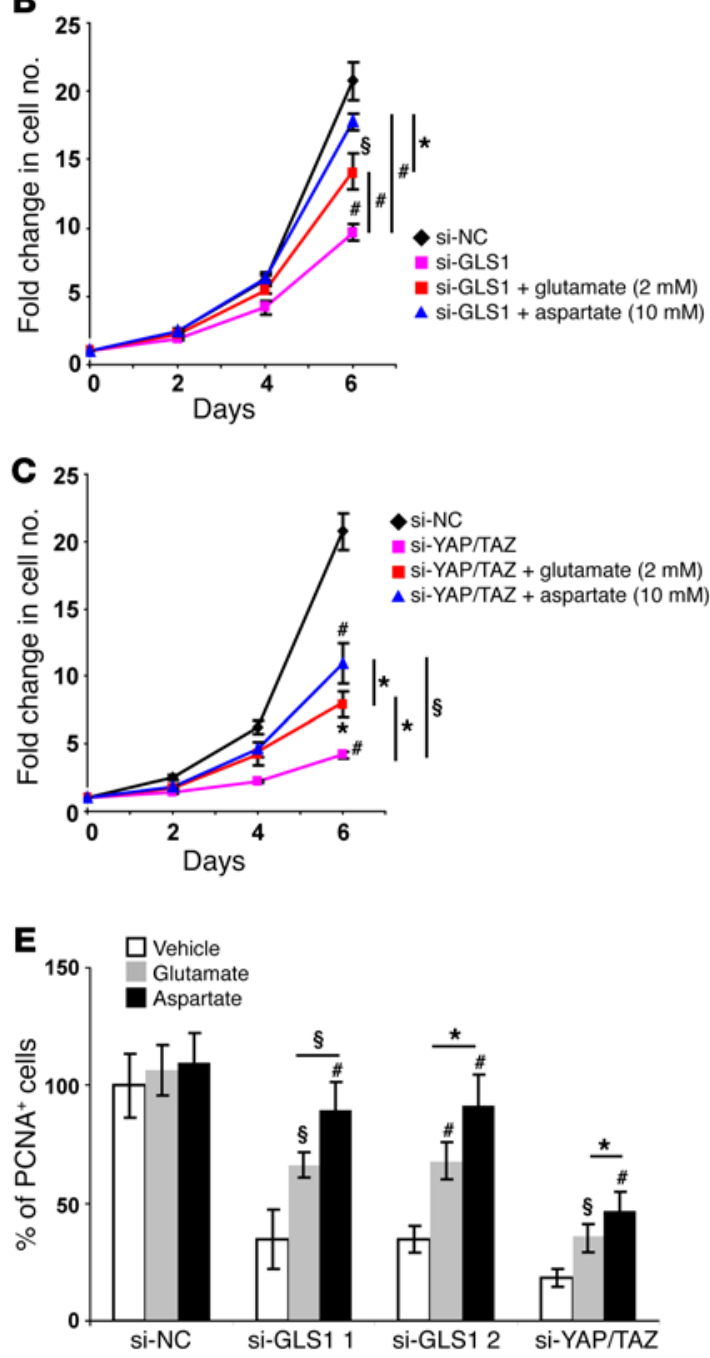

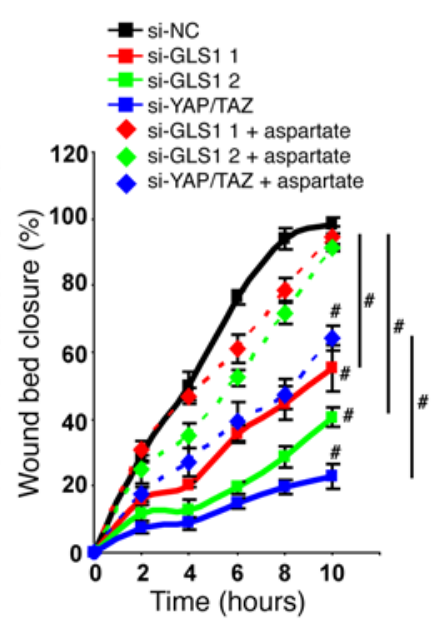


Figure 6. The production of aspartate and glutamate is crucial for YAP/ TAZ-dependent glutaminolytic control of proliferation on stiff matrix. (A) Representative micrographs of PAECs are shown, cultivated on stiff matrix and exposed to the indicated conditions. Cell counting (B and C) and quantification of PCNA fluorescent labeling ( $\mathbf{D}$ and $\mathbf{E}$ ) revealed that siRNA knockdown of GLS1 or YAP/TAZ decreased cell proliferation. Supplementation by glutamate and, to a better extent, aspartate sustained proliferation of PAECs even when GLS1 or YAP/TAZ was knocked down. (F) Similarly, scratch assay demonstrated that aspartate supplementation of PAECs in stiff matrix increased cell migration even during GLS1 or YAP/ TAZ knockdown. In all panels, mean expression in control groups (si-NC) was assigned a fold change of 1 , to which relevant samples were compared. Data are expressed as the mean \pm SD $\left({ }^{*} P<0.05,{ }^{\S} P<0.01,{ }^{\#} P<0.001\right)$ of at least 3 independent experiments performed in triplicate. $P$ values were derived from 1-way ANOVA with a post-hoc Tukey's test. Scale bars: 200 $\mu \mathrm{m}$. See also Supplemental Figure 5.

The YAP/TAZ-GLS1 axis activates glycolysis and glutaminolysis in PAECs and PASMCs exposed to vascular stiffness in rodent and human instances of $P H$ in vivo. In a rat model of inflammatory PAH (monocrotaline-induced), we recently described pulmonary arterial stiffening as an early pathologic event accompanied by increased YAP/TAZ expression in diseased pulmonary arterioles (1). In this same monocrotaline rat model (Figure 7 and Supplemental Figures 6-8), we investigated whether glutaminolysis was activated and correlated with pulmonary arterial stiffness, YAP activation, and PAH. As we previously reported (1), Picrosirius red staining demonstrated an increase in fibrillar collagen deposition in diseased pulmonary arterioles derived from monocrotalineexposed rats (Supplemental Figure 6) and correlated with increased pulmonary arteriolar stiffness, as demonstrated by atomic force microscopy (Figure 7B). These changes were accompanied by hemodynamic manifestations of PAH (Supplemental Figure 7A).

From these rats, $\mathrm{CD} 31^{+}$endothelial cells were isolated from lungs 3 weeks after exposure to vehicle or monocrotaline (Figure $7 \mathrm{~A}$ ), and metabolites were quantified by LC-MS/MS (Figure 7, C-E, and Supplemental Figure 6, C-F). Consistent with our observations of anaplerosis in cultured PAECs grown on stiff matrix (Figure 1), glutamine was decreased (Figure 7C), and aspartate was increased (Figure 7D) in PAH CD31+ cells. Notably, no significant change in glutamate concentration was observed in these cells (Supplemental Figure 6C), which may suggest an elevated glutamate turnover in pulmonary cells in vivo. A decrease of succinate (Supplemental Figure 6F) was also observed, indicative of a decrease in TCA cycle activity, and a glycolytic increase of the lactate/pyruvate ratio (Figure 7E) was present. Consistent with such metabolite alterations, a significant increase of GLS1 expression in $\mathrm{CD} 31^{+}$cells was observed (Figure $7 \mathrm{~F}$ ). Immunoblotting also demonstrated a corresponding increase of GLS1, LDHA, and PC expression at the protein level in both $\mathrm{CD} 31^{+}$(Figure 7, G and $\mathrm{H}$ ) and $\mathrm{CD} 31^{-}$cells from monocrotaline rat lungs (Supplemental Figure 6, H and I). In situ, confocal immunofluorescent microscopy revealed increased GLS1 (Figure 7I and Supplemental Figure 6G), PC, and LDHA staining (Supplemental Figure 6, J and K) in both $\mathrm{CD}^{2} 1^{+}$(endothelial) and $\alpha-\mathrm{SMA}^{+}$(smooth muscle) compartments of diseased pulmonary arterioles. Notably, upregulation of GLS1 (Figure 7I), LDHA (Supplemental Figure 6J), and PC (Supplemental Figure 6K) all correlated with increases of YAP nuclear local- ization and resultant upregulation of $\mathrm{PCNA}{ }^{+}$or $\mathrm{Ki}-67^{+}$proliferating cells in diseased pulmonary arterioles (Figure $7 \mathrm{~J}$ and Supplemental Figure 7, A and B).

In order to determine the precise kinetics of these events during disease progression, we performed in situ confocal immunofluorescent microscopy at various stages of monocrotaline-induced PH in rats (Supplemental Figure 7). Consistent with previous theories of endothelial apoptosis in PH (22), we found an early yet temporary induction of endothelial apoptosis as reflected by cleaved caspase- 3 in situ staining and by caspase $-3 / 7$ activity (days 0-3 after monocrotaline injection) (Supplemental Figure 7, C-E). This was followed by a subsequent decrease of apoptosis and an increase of smooth muscle and endothelial cell proliferation (Supplemental Figure 7, A and B), correlating with an increase of both splice isoforms (KGA and GAC) of vascular GLS1 expression (Supplemental Figure 8, A-C). Taken together, and consistent with our in vitro findings, these results demonstrated that, following vascular injury and just after an early wave of endothelial apoptosis, the development of pulmonary vascular stiffness and glutaminolysis follows the same kinetics as the increase of proliferation of diseased endothelial and smooth muscle cells in vivo.

Consequently, we wanted to determine whether glutaminolytic reprogramming is an active process in human PAH to sustain pulmonary vascular cell proliferation. We studied a cohort of human patients with PAH $(n=13)$ stemming from causes ranging from idiopathic and hereditary etiologies to scleroderma (Supplemental Table 2), as compared with non-PAH subjects $(n=6)$ who died from traumatic or unrelated causes (1). Correlating with increased periarteriolar collagen remodeling in $\mathrm{PAH}$ cases (Figure 8A), a concurrent upregulation of GLS1 (Figure 8, $\mathrm{B}$ and $\mathrm{C}$ ), PC, and LDHA (Supplemental Figure 9, A and B) was observed in both $\mathrm{CD}_{3} 1^{+}$(endothelial) and $\alpha-\mathrm{SMA}^{+}$(smooth muscle) cells (Figure 8B). As with PAH rats (Supplemental Figure 8), both isoforms of GLS1 (KGA and GAC) were increased (Supplemental Figure 9, C and D). GLS1 was increased simultaneously with YAP nuclear localization (Figure 8C), and YAP nuclear localization correlated with increased $\mathrm{PCNA}^{+}$or $\mathrm{Ki}-67^{+}$proliferating vascular cells (Figure 8D and Supplemental Figure 9E). Similar observations were made in plexiform lesions (Supplemental Figure 10, A and B) - late-stage vascular lesions where active proliferation and quiescent apoptosis were consistently observed (Supplemental Figure 10C). Importantly, alterations of these metabolic enzymes correlated with metabolite profiles in circulating plasma, as assessed by LC-MS/MS in samples originating from the main pulmonary artery of $\mathrm{PH}$ individuals (mean pulmonary arterial pressure $[\mathrm{mPAP}] \geq 25 \mathrm{mmHg}$; patient demographic information in Supplemental Table 3). In subjects with particularly high pulmonary arterial pressures (mean pulmonary pressure $>45 \mathrm{mmHg}$ ), lactate/pyruvate ratio was elevated reflective of increased glycolysis, while glutamine/glutamate ratio was decreased and aspartate was increased, indicative of upregulated glutaminolysis and anaplerosis, in comparison with non-PH individuals (mPAP $<25 \mathrm{mmHg}$; Figure $8, \mathrm{E}-\mathrm{G}$ ). Together, these results support the notion that vascular stiffening activates YAP/TAZ in order to induce a glutaminolytic metabolic switch and vascular proliferation in PAH across both rodent and human instances of disease in vivo. 
A Vehicle $(N=6)$ or

monocrotaline

$(60 \mathrm{mg} / \mathrm{kg} ; N=6)$

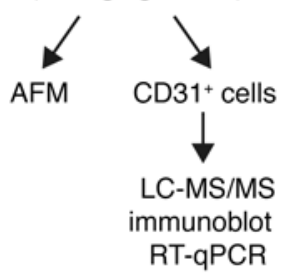

E

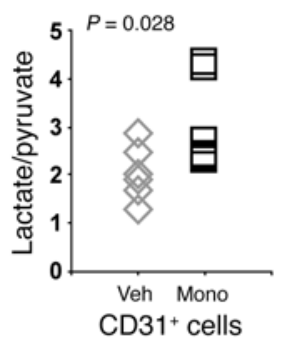

I
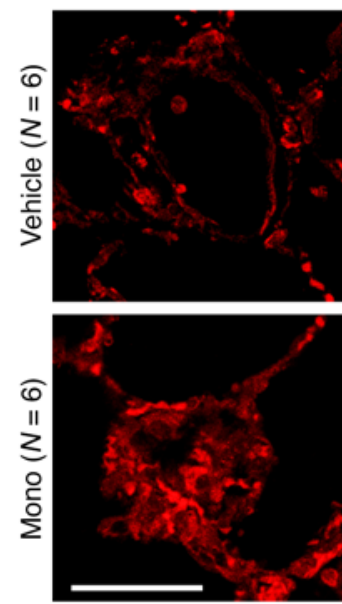

J

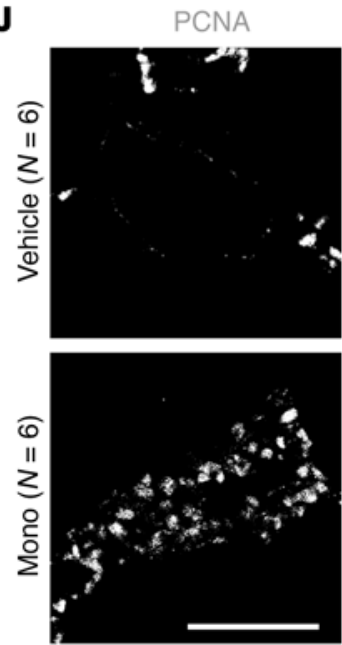

B
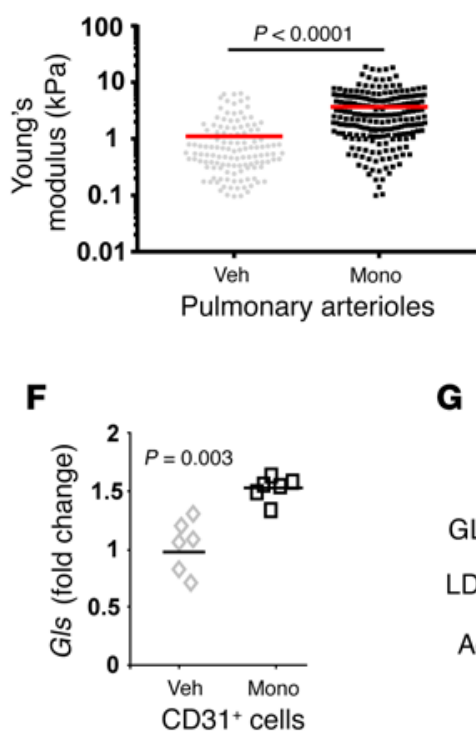

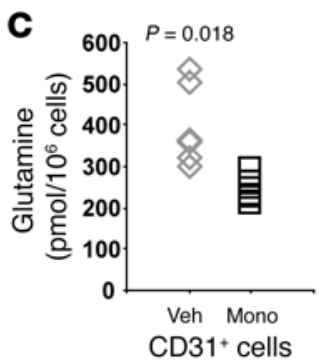

G

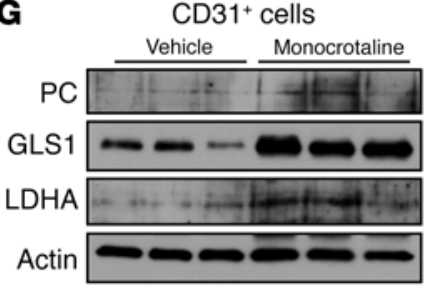

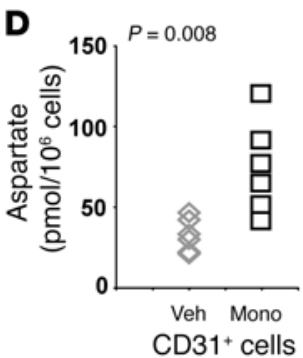

H

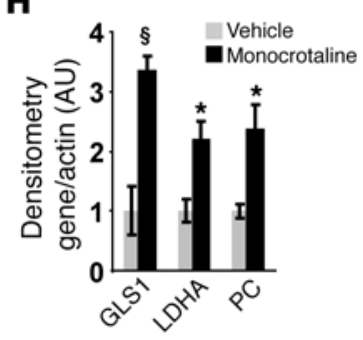

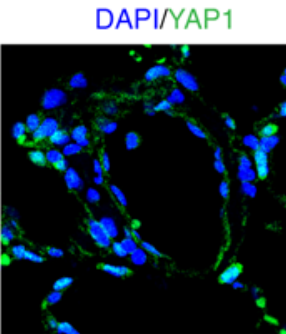

DAPI/GLS1/YAP1
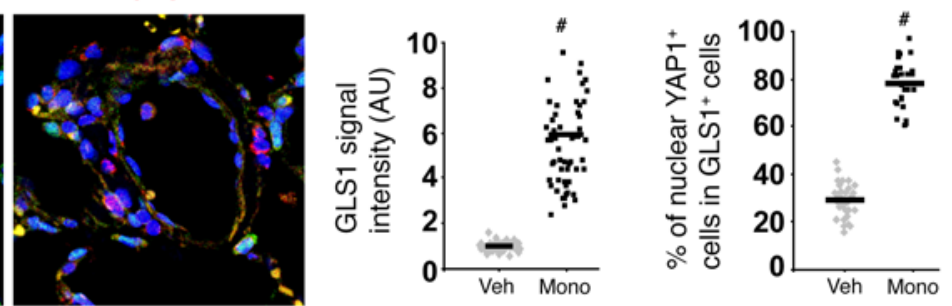
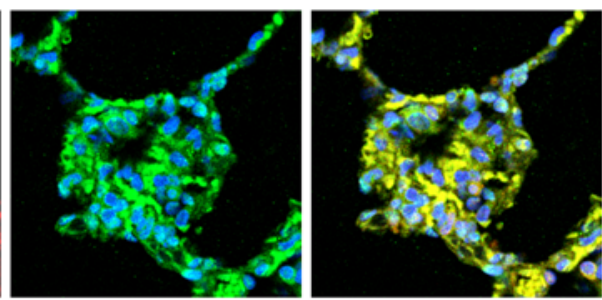

YAP1

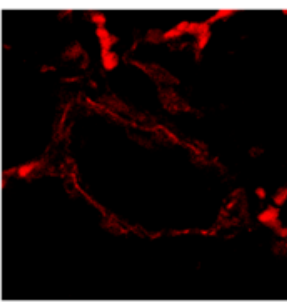

DAPI/PCNA/YAP
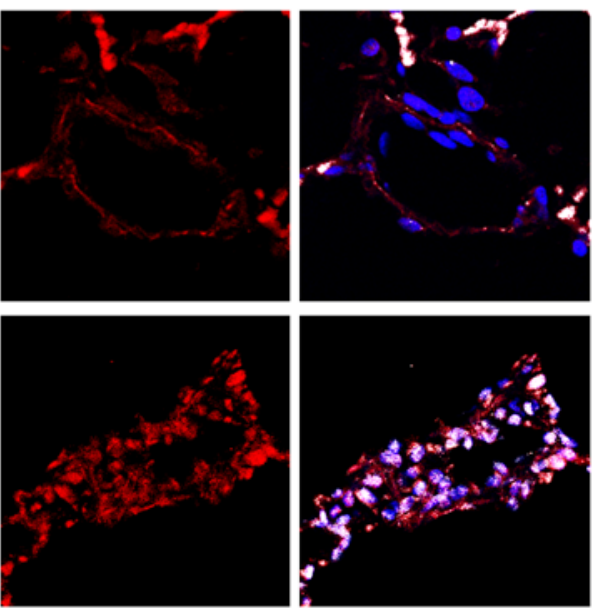

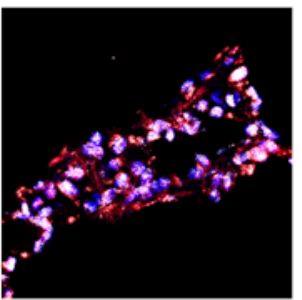

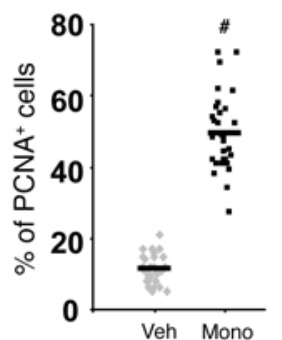


Figure 7. Periarteriolar fibrillar collagen correlates with increased arteriolar stiffness, GLS1, glutaminolysis, and aspartate in monocrotalineexposed PAH rats. Rats were administered $60 \mathrm{mg} / \mathrm{kg}$ of vehicle $(n=6)$ or monocrotaline $(n=6)$ to induce PAH. (A) After 3 weeks, CD31+ endothelial cells were isolated from whole lung for analysis by LC-MS/MS, immunoblot, and RT-qPCR. AFM, atomic force microscopy. (B) AFM revealed increased pulmonary arteriolar ( $<100 \mu \mathrm{m}$ diameter, 5-9 vessels per rat) stiffness in PH lung ( $n=4$ rats) versus untreated ( $n=4$ rats); horizontal lines denote median; symbols denote individual pulmonary arteriolar measurements. $P$ value was calculated by Mann-Whitney $U$ test. (C-E) PAH CD $31^{+}$cells displayed a decrease in glutamine (C) and converse increase in aspartate (D). This correlated with an increase in lactate/pyruvate ratio (E). (F) RT-qPCR of PAH CD31+ cells revealed an increase of Gls expression. (G and $\mathbf{H})$ Immunoblot analysis $(\mathbf{C})$ and densitometry $(\mathbf{H})$ revealed an increase of GLS1, LDHA, and PC in CD31+ lung cells of PAH rats ( $n=3$ rats per group). (I and J) Coimmunofluorescence microscopy demonstrated an increase of YAP1/GLS1 (I) and YAP1/PCNA (J) double-positive cells in diseased pulmonary arterioles. In all panels, mean expression in control groups was assigned a fold change of 1 , to which relevant samples were compared. Data are expressed as the mean \pm SD $\left({ }^{*} P<0.05,{ }^{\circledR} P<0.01\right.$, $\left.{ }^{\#} P<0.001\right)$. In all panels except B, $P$ values were derived from 2-tailed Student's $t$ test. Scale bars: $50 \mu \mathrm{m}$. See also Supplemental Figures 6-8.

The YAP/TAZ-GLS axis induces glycolysis and glutaminolysis in primates with SIV-PAH and in people with HIV-induced PAH. Because rodent models of $\mathrm{PAH}$ do not replicate all aspects of disease in humans, we wanted to determine whether this same molecular axis is active in a more relevant model organism without the use of a direct hypoxic stimulus. Previously, a nonhuman primate model of HIV-induced PAH was described in rhesus macaques infected with SIV (23). Importantly, such a model replicates the hemodynamic and histologic manifestations of PAH. It also displays an incomplete penetrance with $50 \%$ to $60 \%$ of infected macaques developing $\mathrm{PAH}$, thus consistent with the incomplete penetrance of PAH with HIV infection in humans (24). Importantly, similar to the monocrotaline-exposed rats (Figure 7), in a cohort of SIV-infected macaques with confirmed hemodynamic (Figure 9A) and histologic (Supplemental Figure 11A) manifestations of $\mathrm{PAH}$, Picrosirius red staining demonstrated an increase of periarteriolar fibrillar collagen (Figure 9B) as compared with that in non-PAH, SIV-infected animals. Diseased pulmonary arterioles in SIV-PAH macaques also displayed increased expression of both GLS1 isoforms (Figure 9C and Supplemental Figure 11, B-D), PC, and LDHA (Supplemental Figure 11, F and G), correlating with increased YAP nuclear localization (Figure 9C and Supplemental Figure 11, F and G), proliferating $\mathrm{PCNA}^{+}$or $\mathrm{Ki}-67^{+}$cells (Figure 9D and Supplemental Figure 11E), and nonapoptotic, cleaved caspase-3-negative cells (Supplemental Figure 11E).

Finally, on the basis of these findings in SIV-PAH macaques, we wanted to determine whether humans suffering from HIVPAH may also display signs of increased pulmonary vascular stiffness and consequent alterations in vascular glycolysis and glutaminolysis. We studied a cohort of $42 \mathrm{HIV}$-infected individuals who underwent pulmonary arterial catheterization, leading to a diagnosis of PAH in 11 individuals (Supplemental Table 4). Analysis of invasive hemodynamic data of HIV-PAH subjects revealed a significant decrease of pulmonary arterial compliance (Figure 10A) consistent with an increase of pulmonary artery stiffness in comparison with HIV-infected, non-PAH individuals. Important- ly, by quantification of peripheral venous plasma metabolites from a separate cohort of HIV-infected people with and without PAH (Supplemental Table 5), an increase of lactate/pyruvate ratio was observed (Figure 10B), indicative of increased glycolytic activity, while a decrease in glutamine/glutamate ratio and an increase in aspartate were consistent with upregulation of glutaminolysis and anaplerosis in HIV-PAH (Figure 10, C and D). Consequently, mirroring the molecular findings in rodent and other instances of human PAH, these observations of YAP/TAZ-GLS activation in HIV-PAH correlate with the tightly linked connection between pulmonary vascular stiffness and metabolic dysregulation.

Modulation of pulmonary vascular stiffness and YAP/TAZdependent mechanotransduction regulates glutaminolysis and $\mathrm{PH}$ manifestation in vivo. To establish definitively whether periarteriolar ECM remodeling and YAP/TAZ modulate vascular cell metabolism in vivo, we tested whether alteration of YAP/TAZ-dependent mechanotransduction directly controls glutaminolysis and $\mathrm{PH}$ development in the monocrotaline rat model. First, using a known pharmacologic inhibitor ( $\beta$-aminopropionitrile, BAPN) of lysyl oxidase (Lox), the enzyme responsible for collagen cross-linking and consequent matrix stiffening, we determined whether inhibition of ECM stiffening could prevent the metabolic changes and downstream PH manifestations observed in monocrotalineexposed rats (Figure 11A). BAPN treatment decreased pulmonary Lox activity and consequent periarteriolar ECM stiffening, as assessed by atomic force microscopy (Figure 11, C and D). In line with previous reports (25), a trend toward decreased systemic mean arterial pressure was observed with BAPN treatment (Supplemental Figure 12E) but without adverse effects on left ventricular cardiac function or heart rate (Supplemental Figure 12, A-D). Consistent with our in vitro results, reduction of ECM stiffening by BAPN led to a decrease of YAP and GLS1 expression (Figure 12A), YAP-dependent gene expression (Figure 11E), and downstream GLS activity, as reflected by direct enzymatic activity measurement (Figure 11F). Such metabolic effects further decreased vascular endothelial and smooth muscle proliferation, as reflected by in situ arteriolar staining (Figure 12A), and ameliorated hemodynamic and histologic manifestations of $\mathrm{PH}$, as measured by vascular remodeling and muscularization (Figure 12A), and right ventricular systolic pressure (RVSP) (Figure 12B).

In a parallel experiment, verteporfin, a known pharmacologic inhibitor of YAP (26) previously used to study YAP activity in tumorigenesis in vivo (27), was used to interrogate whether YAP is also essential for activating vascular glutaminolysis and $\mathrm{PH}$ in monocrotaline-injected rats (Figure 11B). As expected, verteporfin decreased YAP-dependent gene expression (Figure 11E) without adverse effects on left ventricular cardiac function, heart rate, or systemic blood pressure (Supplemental Figure 12). Consequently, in a similar fashion to BAPN but to a much more robust degree, verteporfin improved the downstream metabolic (GLS1 expression and GLS activity; Figure 11, E and F), proliferative (Figure 12A), and end-stage manifestations of $\mathrm{PH}$, including reductions in vascular remodeling/muscularization, RVSP, and right ventricular remodeling (Fulton index) (Figure 12, A-C). Notably, verteporfin also decreased pulmonary arteriolar stiffness (Figure 11D), consistent with our prior report of YAP-dependent control of ECM remodeling (1). As a result, these data provide causative 
A Picrosirius red Picrosirius red
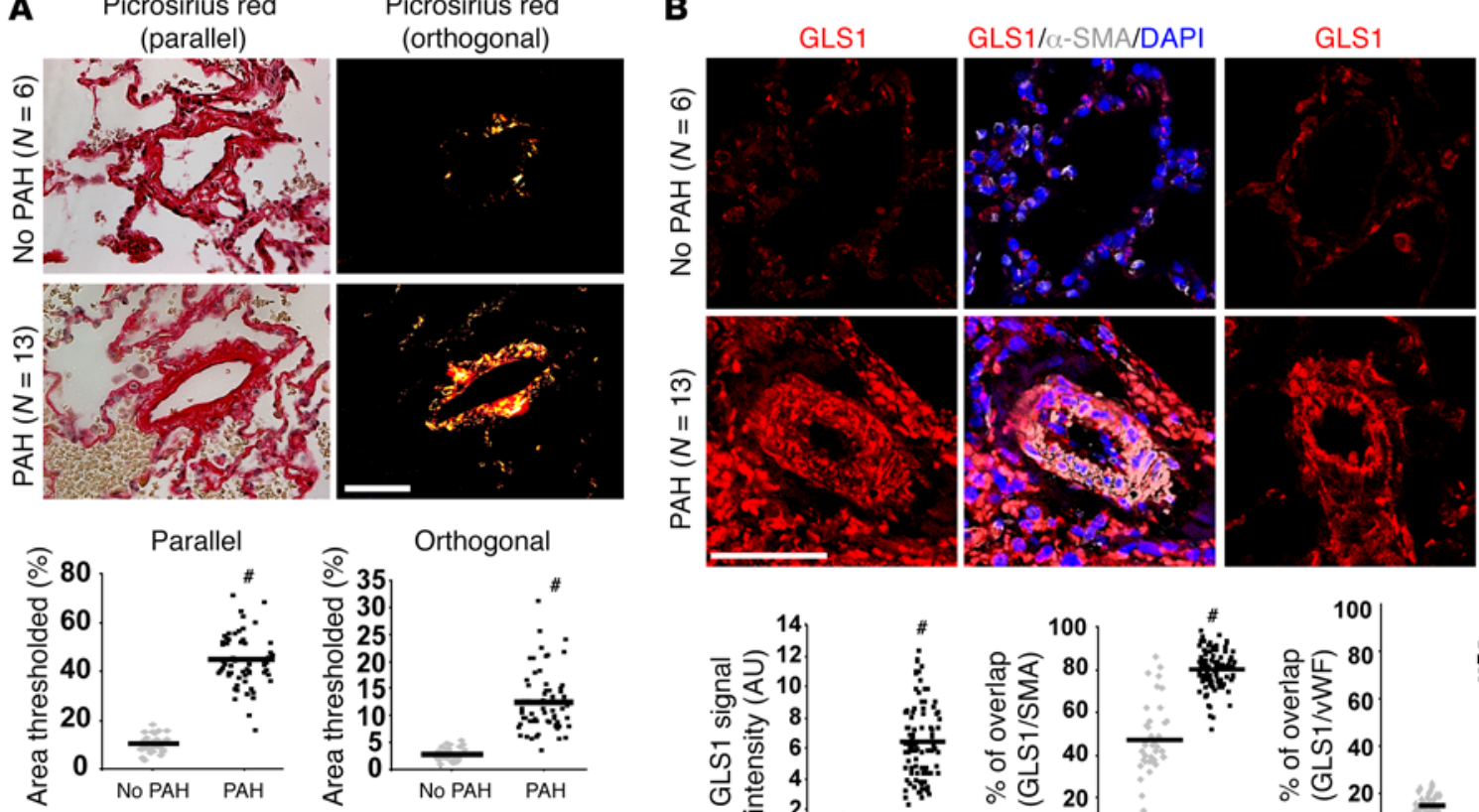

GLS1/NWF/DAPI

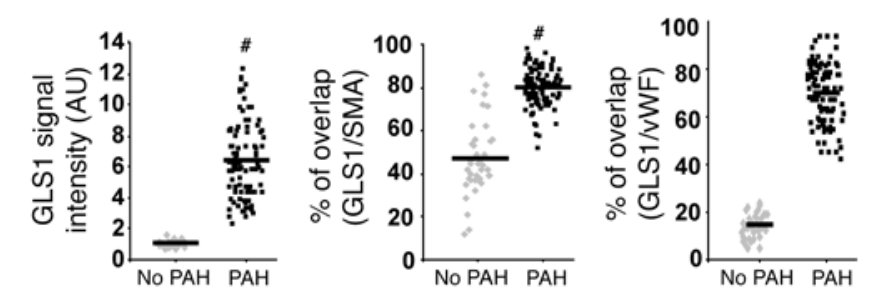

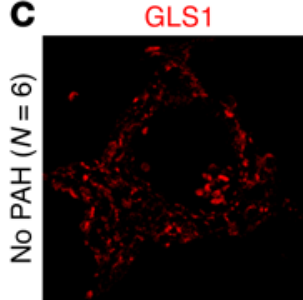
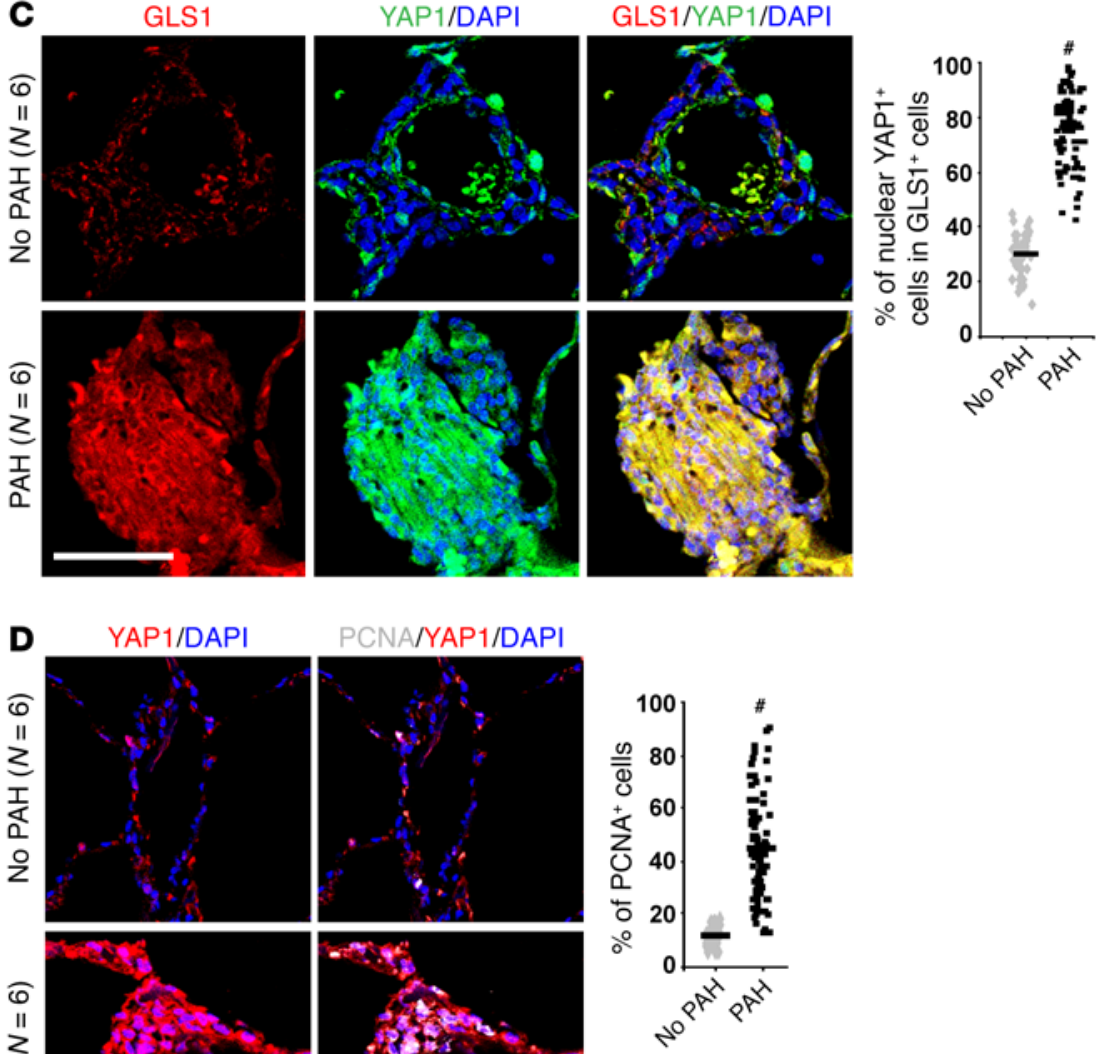

E
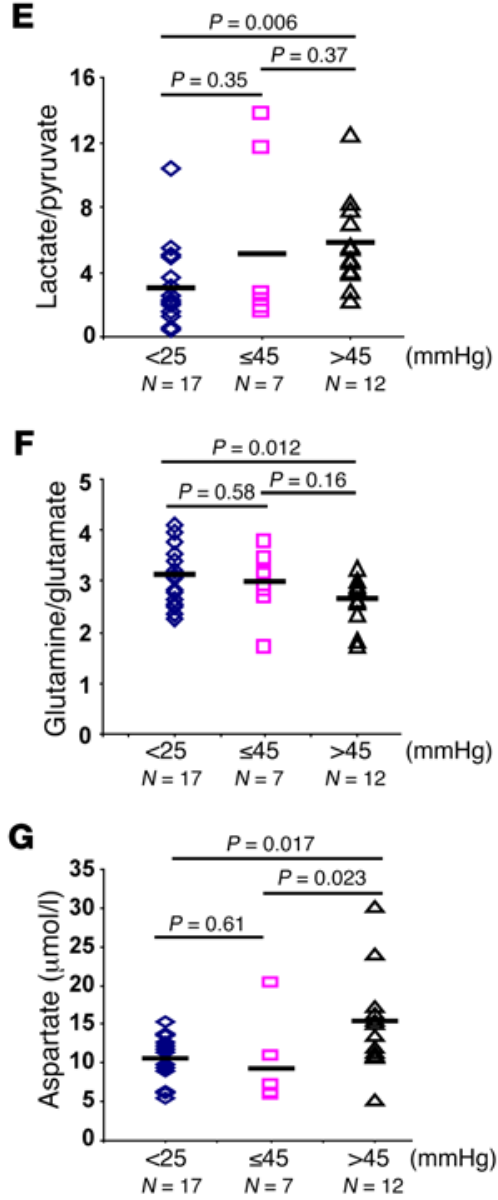
Figure 8. Periarteriolar fibrillar collagen correlates with increased GLS1, glutaminolysis, and aspartate in multiple forms of human PAH. (A) By Picrosirius red stain of human PAH $(n=13)$ versus non-PAH lung $(n=6)$, quantification of less-than-100- $\mu$ m vessels ( 5 vessels per patient) confirmed increased collagen deposition and fibrillar collagen assembly in PAH. (B) In human PAH tissue ( $n=13)$, coimmunofluorescence microscopy and quantification also confirmed an increase of GLS1 in both $\alpha-S M A^{+}$and $\mathrm{VWF}^{+}$cells in diseased pulmonary arterioles as compared with controls $(n=6)$. (C and $\mathbf{D})$ Coimmunofluorescence microscopy revealed increased YAP1/CLS1 (C) and YAP1/PCNA (D) double-positive cells in diseased pulmonary arterioles (PAH, $n=6$, vs. no $\mathrm{PAH}, n=6$ ). (E-C) In correlation, targeted LC-MS/MS revealed increasingly higher plasma levels of lactate/ pyruvate ratio (E), lower levels of glutamine/glutamate ratio (F), and increasing levels of aspartate (C) in patients with increasing hemodynamic severity of $\mathrm{PH}$. In all panels, mean expression in control groups was assigned a fold change of 1 , to which relevant samples were compared. Data are expressed as the mean $\pm \mathrm{SD}\left({ }^{*} P<0.001\right)$. In $\mathbf{A}-\mathbf{D}, P$ values were derived from 2-tailed Student's $t$ test. In E-G, $P$ values were derived from 1-way ANOVA with a post-hoc Tukey's test. Scale bars: $50 \mu \mathrm{m}$. See also Supplemental Figures 9 and 10.

evidence in vivo that ECM stiffening relies on YAP/TAZ-specific mechanotransduction in order to induce pulmonary vascular glutaminolysis and anaplerosis, proliferation, and $\mathrm{PH}$.

GLS1-dependent inhibition of glutaminolysis decreases pulmonary vascular cell proliferation in vivo and ameliorates $P H$. Finally, to investigate whether glutaminolysis itself is essential for promoting pulmonary vascular proliferation in $\mathrm{PH}$, we administered 2 separate pharmacologic inhibitors of GLS1 - C968 (19) and CB-839 (28) - in monocrotaline-exposed rats using either a disease prevention (Figure 13A) or a disease reversal (Figure 13B) dosing protocol. In both cases, C968 and CB-839 treatments decreased GLS activity in whole rat lung as compared with control (Figure 13, C and D) without adverse effects on left ventricular function, heart rate, or systemic blood pressure (Supplemental Figure 13). Correspondingly, $\mathrm{C} 968$ and $\mathrm{CB}-839$ both decreased the presence of proliferation markers $\left(\mathrm{PCNA}^{+}\right.$or Ki- $67^{+}$) in $\mathrm{CD}^{2} 1^{+}$or $\mathrm{vWF}^{+}$(endothelial) and $\alpha-\mathrm{SMA}^{+}$(smooth muscle) pulmonary arteriolar cells in comparison with control PH rats (Figure 13, E-H) without effect on pulmonary arteriolar cell apoptosis (Supplemental Figure 14). As a result, both C968 and CB-839 significantly decreased pulmonary arteriolar remodeling (Figure 14, A and B) and muscularization (Figure 14, C and D), RVSP (Figure 14, E and G), and right ventricular remodeling (Figure 14, $\mathrm{F}$ and $\mathrm{H}$ ). Taken together, these results directly implicate GLS1 and glutaminolysis, a process dependent on ECM stiffening, as critical metabolic mediators necessary for sustaining pulmonary vascular proliferation in $\mathrm{PH}$.

\section{Discussion}

Both pulmonary vascular stiffness and metabolic derangements have been described as crucial mediators of the pro-proliferative condition in PH. Yet their precise mechanistic connections had not been defined until now. In this study, we elucidated the crucial connection of YAP/TAZ mechanoactivation to the glutaminolytic enzyme GLS1 and showed that they are required for coordination of the cellular energetic needs for proliferation in the setting of aerobic glycolysis (Figure 15). Such molecular insights advance the paradigm of vascular stiffness beyond merely the study of hemodynamic effects on vascular compliance, but revealing it as a specific metabolic cause of vascular remodeling and PH development. These results also alter our fundamental understanding of the dysregulated metabolic axis in $\mathrm{PH}$ itself beyond direct hypoxic injury by revealing both glutamine metabolism and aerobic glycolysis as integrally linked through a shared hierarchy of regulation via YAP/TAZ. Finally, by placing glutaminolysis as a central mechanism of how the extracellular environment dictates pulmonary vascular dysfunction, these results form a basis for developing novel therapeutics, or even more likely, repurposing already approved medications, that target the YAP/TAZ-GLS1 axis in PH.

Recent work has advanced the concept that vascular stiffening in $\mathrm{PH}$ is an early and potent pathogenic trigger in $\mathrm{PH}(1,3)$. Yet, beyond the association with vascular proliferation, a detailed characterization has been missing of downstream metabolic pathways affected by such mechanical stimuli. Here, the identification of glutaminolysis as a mechanoactivated process coregulated with aerobic glycolysis advances our understanding of the regulatory hierarchy seen in the metabolic reprogramming in PH. Such an interface between stiffness and metabolism draws parallels to related reprogramming events proposed in tumors in relation to matrix remodeling (29). By its direct causative relation to metabolic dysregulation, it also reinforces the paradigm of vascular stiffness as an initiating pathogenic trigger of this disease rather than merely an end-stage feature. This model of a stiffnessmetabolism interface in $\mathrm{PH}$ now invokes intriguing questions regarding the exact timing of these molecular events in a variety of PH subtypes and whether these events can be detected clinically and prevented before disease advancement. It also raises a question of how the metabolic response to vascular stiffness may relate to other metabolic regulators in PH that are already known to be driven by hypoxia, such as pyruvate dehydrogenase kinase (30), miR-210 (31), and the bone morphogenetic protein receptor type 2 (BMPR2) (8), among others.

Elucidation of a connection linking vessel stiffness with glutaminolysis and proliferation also provides fundamental insight into the interplay between cellular proliferation, migration, and apoptosis among multiple vascular cell types during $\mathrm{PH}$ development. As described in other contexts (32), increased glutaminolysis and anaplerosis in response to stiff matrix and YAP/TAZ activation answer a key metabolic need to sustain the hyperproliferative state, particularly in PASMCs, and thus drive vascular remodeling in PH. Separately, in adventitial fibroblasts, we recently described a YAP/TAZ-miR-130/301 feedback loop whereby matrix stiffening spreads through pulmonary vasculature and perhaps even pulmonary parenchyma via mechanoactivation of naive fibroblasts that contact stiffened matrix (1). Given the current findings implicating YAP/TAZ activation with glutaminolysis, it is possible that glutaminolysis and anaplerosis in fibroblasts are also inherently linked to the control of matrix stiffening and remodeling.

Moreover, vessel stiffness and glutaminolysis prominently modulate the still incompletely defined dysfunction of PAECs in PH. Historically, the results of using single markers of proliferation or endothelial cell lineage to analyze diseased pulmonary vessels have been challenging to interpret. To address those concerns, our methods relied on multiple independent markers of endothelial lineage (CD31 and vWF) and proliferation (Ki-67 and PCNA) to 
A

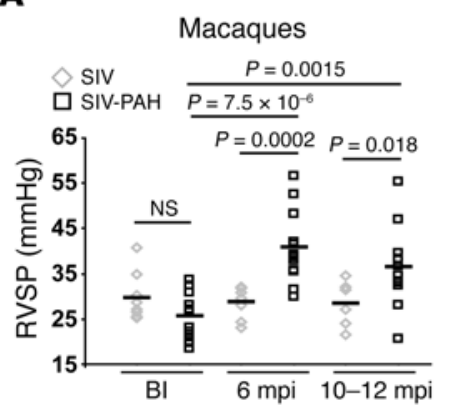

B

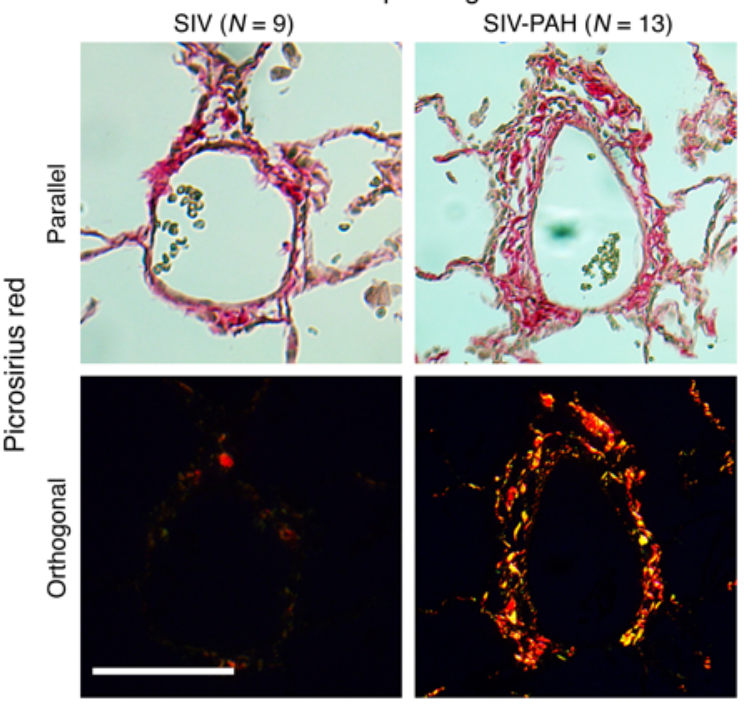

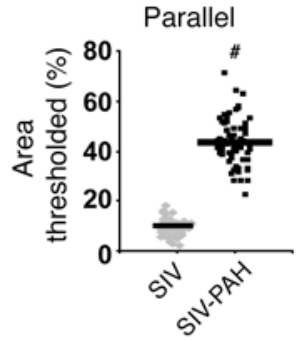

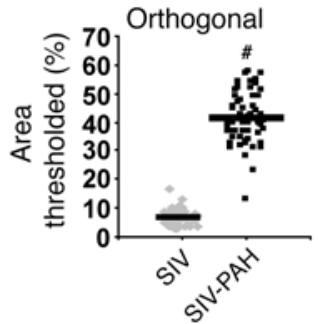

C

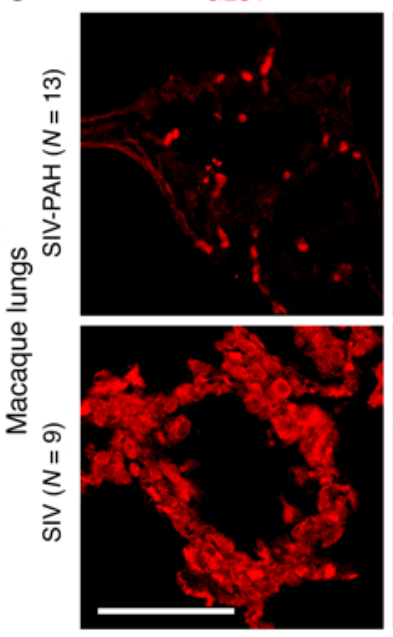

DAPI/YAP1
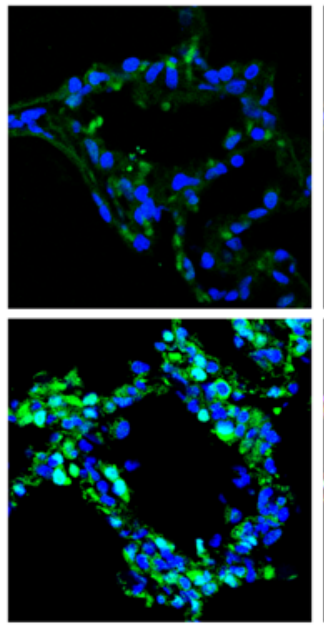

DAPI/GLS1/YAP1
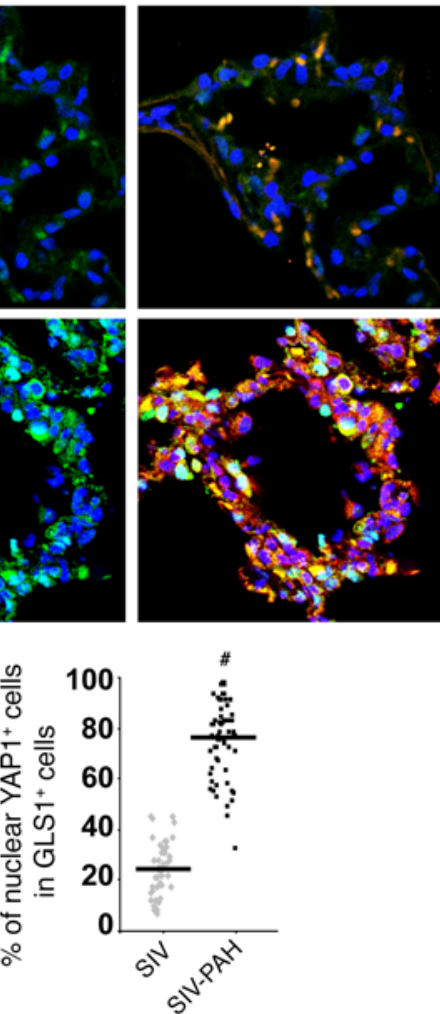

5 (")
D $\quad \operatorname{siv}(N=9)$
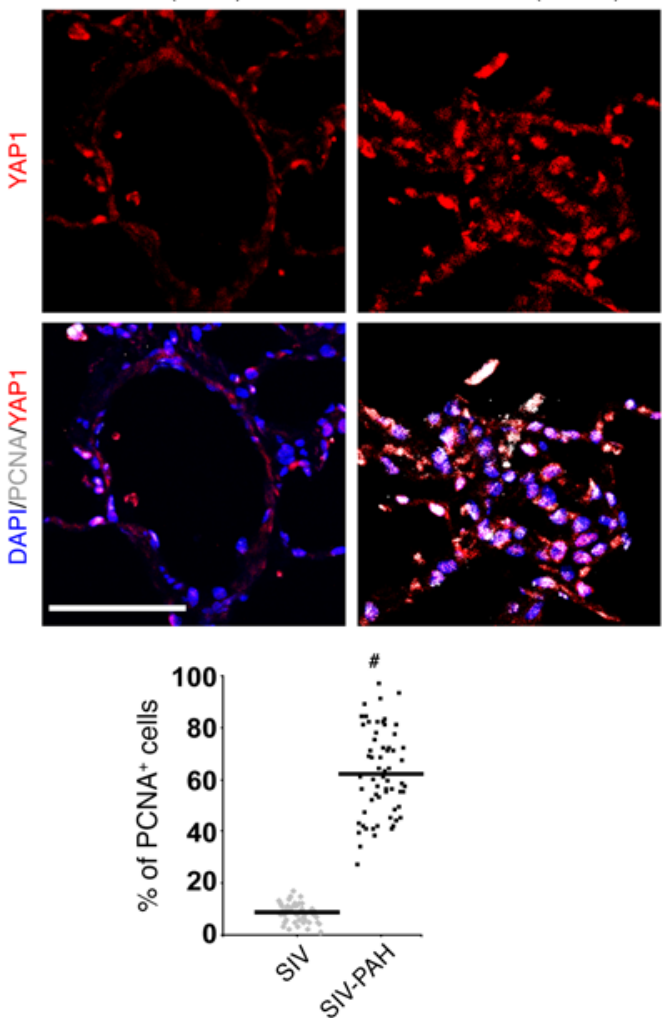

SIV-PAH $(N=13)$

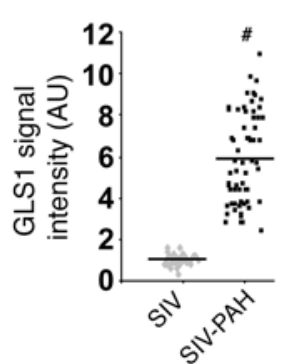

Figure 9. Periarteriolar fibrillar collagen deposition correlates with increased GLS1, glutaminolysis, and aspartate production in primate examples of SIV-induced PAH. (A-D) Rhesus macaques were infected with SIV and evaluated over the course of 6 and 10-12 months postinfection (mpi) as compared with before infection (BI). (A) In 13 of the 22 macaques infected by SIV, PAH was evident as assessed by RVSP and histologic remodeling (see Supplemental Figure 11A). (B) Picrosirius red stain revealed increased periarteriolar fibrillar collagen deposition in SIV-PAH. (C and D) Coimmunofluorescence microscopy revealed an increase of YAP1/GLS1 (C) and YAP1/PCNA (D) double-positive cells in diseased pulmonary arterioles. In all panels, mean expression in control groups was assigned a fold change of 1 , to which relevant samples were compared. $P$ values were derived from 1-way ANOVA and post-hoc Tukey's tests in A, while 2-tailed Student's $t$ test was used for other comparisons ( $\left.{ }^{\#}<0.001\right)$. Scale bars: $50 \mu \mathrm{m}$. See also Supplemental Figure 11. 

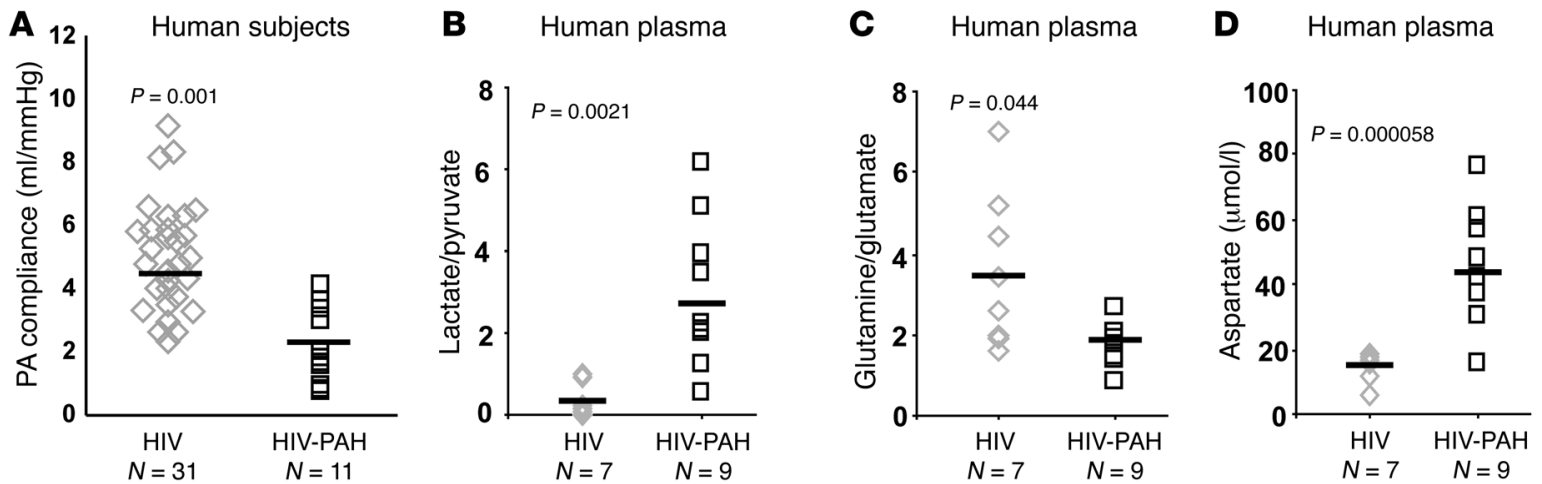

\begin{abstract}
Figure 10. Pulmonary arterial compliance is inversely correlated with increased glutaminolysis and aspartate production in human examples of HIVinduced PAH. (A) By invasive pulmonary arterial catheterization, decreased pulmonary arterial (PA) compliance was observed in a cohort of documented HIV-PAH patients $(n=11)$ as compared with HIV-infected patients alone $(n=31)$. (B-D) In correlation, targeted LC-MS/MS in plasma revealed increased lactate/pyruvate ratio (B), decreased glutamine/glutamate ratio (C), and increased aspartate (D) in an independent HIV-PAH patient cohort $(n=9)$ as compared with HIV-infected patients alone $(n=7)$. In all panels, mean expression in control groups was assigned a fold change of 1 , to which relevant samples were compared. $P$ values were derived from a 2-tailed Student's $t$ test.
\end{abstract}

establish our conclusions. In doing so, our findings are consistent with the model of spatiotemporal balance of PAEC apoptosis and proliferation, originally described by Voelkel and colleagues (33) and echoed by others (34). We found that YAP/TAZ-GLS1 activation, glutaminolyis, and proliferation in PAECs closely followed an initiating wave of injury and PAEC apoptosis. Such proliferation may feed into increased PAEC turnover at earlier stages of $\mathrm{PH}$ (i.e., days 3-7 after monocrotaline injection in rats; Supplemental Figure 7) at which a balance of PAEC apoptosis and proliferation was discerned. However, during later stages of $\mathrm{PH}$ when upregulation of YAP and GLS1 was persistent, markers of proliferation were also more evident. As reported by others (35), PAEC apoptosis cannot be ruled out at later disease time points. However, our data indicate that these apoptotic events must be occurring in cells other than the prevalent proliferative component driven by vascular stiffness and glutaminolysis (Supplemental Figure 7). This finding correlates with our observations of human plexiform lesions in PAH (Figure 8C and Supplemental Figure 10), where YAP upregulation and glutaminolytic processes accompanied overgrowth of PAEC-like cells. Even in settings where the diseased endothelial layer is not overgrown, it is an intriguing possibility that hyperactivated and glutaminolytic PAECs are reprogrammed for pathophenotypes in addition to proliferation, including endothelial-to-mesenchymal transition - a process that has been directly connected to $\mathrm{PH}(36,37)$ and where further proliferation could allow for endothelial cells to feed into medial hyperplasia. Finally, correlating with increased PAEC proliferation, our findings revealed a promigratory phenotype promoted by matrix stiffness and the YAP-GLS1 axis (Figure 6F). A disorder of proliferation and migration has been observed in human plexiform lesions (22), and more recent studies of hyperproliferative PAECs in $\mathrm{PH}$ have described an accompanying migratory phenotype (38). Such disordered migration may contribute to abnormal angiogenesis in $\mathrm{PH}$ - which in some cases has been linked to a proangiogenic and pathogenic remodeling of the pulmonary arteriole (39) and in other cases has been linked to a deficiency of angiogenesis (39) and a pruning of the entire pulmonary vascular tree. Consequently, our work places vessel stiffness, the YAP/TAZ-GLS1 axis, and gluta- minolysis at central points affecting multiple pulmonary vascular cell phenotypes in a timed and stage-specific manner. Accordingly, future work should delineate connections of vascular stiffness and glutaminolysis with pulmonary vascular cell reprogramming, crosstalk, and timed evolution of cellular function and cellular identity in this disease.

The mechanoactivation of YAP/TAZ as a central mediator of glutaminolysis also advances our understanding of the intricate control of metabolism by Hippo signaling. Notably, our data indicated that YAP and TAZ together, but not alone (data not shown), are necessary for GLS1, LDHA, and PC upregulation (Figure 3, $\mathrm{C}-\mathrm{E}$ ), at least for the in vitro endogenous response to matrix stiffening. Thus, there exists some degree of compensation between YAP and TAZ allowing for homeostatic maintenance of the metabolic phenotype even when 1 factor is repressed. The dynamics of this compensatory relationship, however, await further mechanistic definition in vivo. Moreover, cellular energy status has been implicated as a potent regulator of YAP/TAZ activity either through AMP-kinase activation $(9,10)$, induction of aerobic glycolysis (40), or mevalonate metabolism (41). A connection between YAP activity and glutamine synthetase (GS) has been reported in the liver, where GS expression can predominate $(42,43)$. Yet, in other tissue compartments such as the pulmonary vasculature, our findings more directly define these factors as mechanical sensors to reprogram glycolytic and glutaminolytic pathways and coordinate with cellular proliferation. This reciprocity among YAP/TAZ and metabolic cues suggests an adjustable, feedback-driven pathway and may be partly responsible for individualized "tuning" of the metabolic program, depending on burden of ECM remodeling, PH subtype, severity, or temporal stage. Furthermore, it is likely that the metabolic actions of Hippo signaling extend to an even wider sphere of influence than vascular stiffness or $\mathrm{PH}$ alone. In the contexts of organ development and tumorigenesis, it is tempting to speculate on the master regulatory role of Hippo signaling in glutaminolysis and glycolysis as a primary mechanism to balance proliferative capacity with efficient energy production.

The identification of glutaminolysis as a crucial mediator of the $\mathrm{PH}$ pathophenotype shifts our attention to essential regulatory 
A

BAPN water $(100 \mathrm{mg} / \mathrm{kg} / \mathrm{d})$

or control water

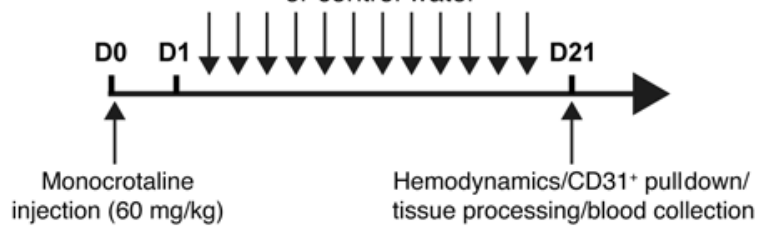

B

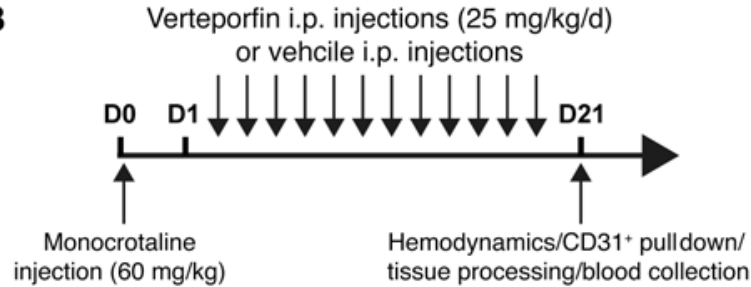

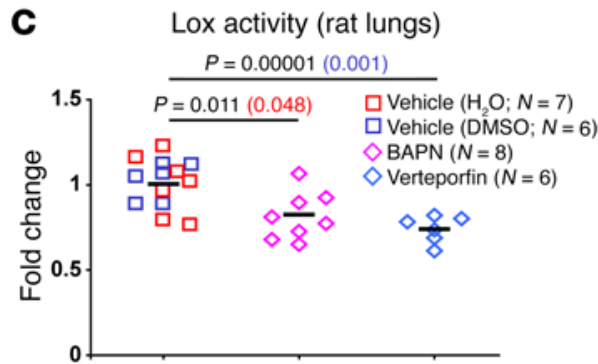

E

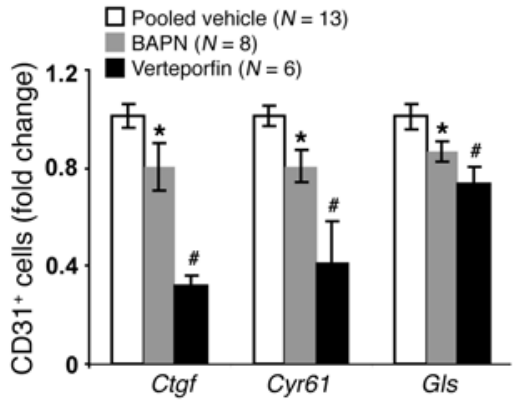

D

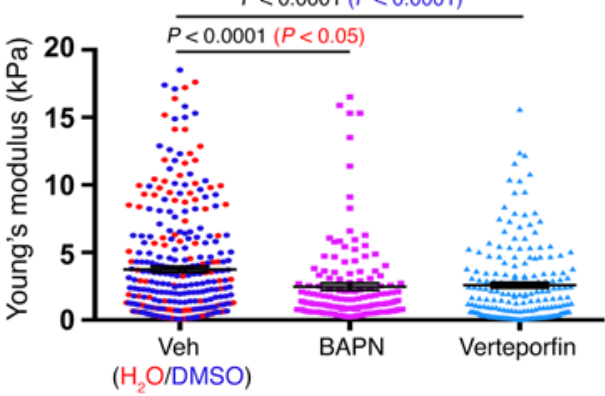

$\mathbf{F}$

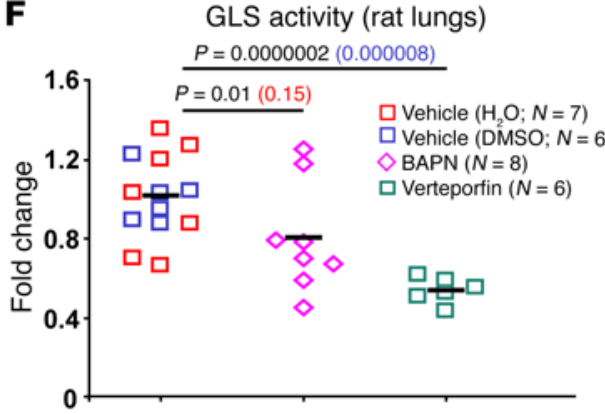

Figure 11. Manipulation of vascular mechanotransduction in vivo controls glutaminolysis. (A and $\mathbf{B})$ Following monocrotaline exposure, rats were treated with daily BAPN $(n=8)$ versus vehicle $(\mathbf{A} ; n=7)$ or with daily i.p. injections of separate verteporfin $(n=6)$ versus separate vehicle $(\mathbf{B} ; n=6)$. (C) Both BAPN and verteporfin decreased Lox activity in lungs of monocrotaline-exposed rats. (D) Atomic force microscopy revealed decreased pulmonary arteriolar (<100 $\mu \mathrm{m}$ diameter) stiffness in BAPN- and verteporfin-treated rats. Horizontal lines denote median; symbols denote individual pulmonary arterial measurements. (E and F) RT-qPCR of PAH CD31+ cells revealed a decrease of 2 YAP-dependent target genes, Ctgf and Cyr61, as well as a decrease of Gls expression (E) and GLS activity (F) in BAPN- and verteporfin-treated rats. Black font reports $P$ values when comparing with pooled vehicle controls; red or blue font reports $\mathrm{P}$ values when comparing with separate vehicle controls. sln all panels, mean expression in control groups was assigned a fold change of 1 , to which relevant samples were compared. $P$ values were derived from 1 -way ANOVA and post-hoc Tukey's tests $\left({ }^{*} P<0.05\right.$, $\left.{ }^{\#} P<0.001\right)$. See also Supplemental Figure 12.

metabolic checkpoints beyond aerobic glycolysis in this disease. A metabolomic screen of pulmonary vascular endothelium independent of hypoxia suggested broad metabolic reprogramming beyond aerobic glycolysis in the context of mutant BMPR2 expression (44), but the points of regulation were not defined. Increased glutamine utilization and metabolism have been observed in the right ventricles of rats and humans with $\mathrm{PH}$ (16). Cardiac glutaminolysis, however, appeared to be driven by microvascular ischemia rather than through mechanosensation. Indeed, the regulation and downstream effects of glutaminolysis independent of hypoxic injury should guide future work to define precisely the crucial steps in reprogramming the $\mathrm{PH}$ metabolic state. As also reported in malignant cells $(14,15)$, we found that aspartate is crucial for stiffnessdependent vascular proliferation (Figure 6). However, all metabolic properties of cancer may not be fully replicated in $\mathrm{PH}$. While evidence exists in tumors of preferential GLS1 isoform activity (45), both isoforms of GLS1 (KGA and GAC) were equally responsive to matrix stiffness in vitro (Supplemental Figure $1 \mathrm{~K}$ ) and in $\mathrm{PH}$ in vivo (Supplemental Figure 8, B and C; Supplemental Figure 9, C and D; and Supplemental Figure 11, C and D), thus arguing against a differential isoform switch in PH. Nonetheless, the identification of GLS1 as a nodal control point emphasizes glutaminolysis and anaplerosis as key molecular determinants underlying the overarching similarity between the pathogenesis of $\mathrm{PH}$ and cancer (46). Moreover, beyond GLS1, 2 additional enzymes - LDHA and PC - were identified here as linked checkpoints in stiffness-mediated alterations of glycolysis and anaplerosis (Figure 1), indicating an even broader level of control over the metabolic landscape in $\mathrm{PH}$.

The mechanistic connection of the YAP/TAZ-GLS1 axis to HIV-PAH also contributes needed insight into the pathogenesis of this enigmatic form of $\mathrm{PH}$. There exists an increased prevalence of PAH in HIV-infected individuals (24), but little is known about the 
A
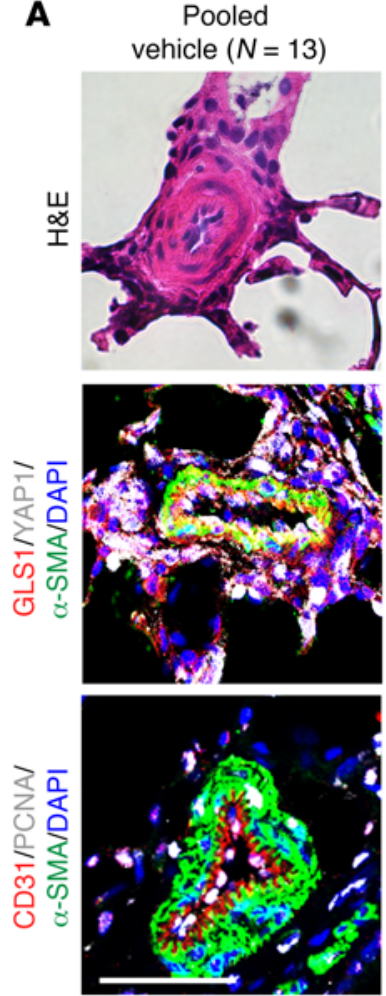
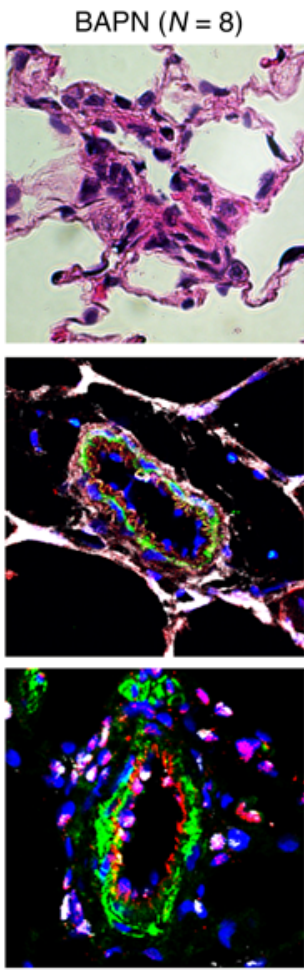
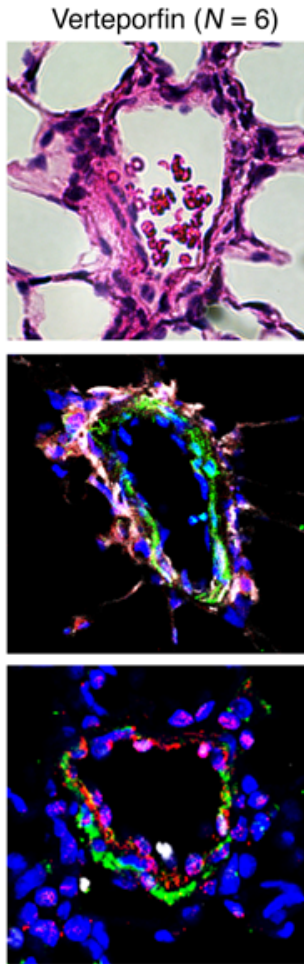
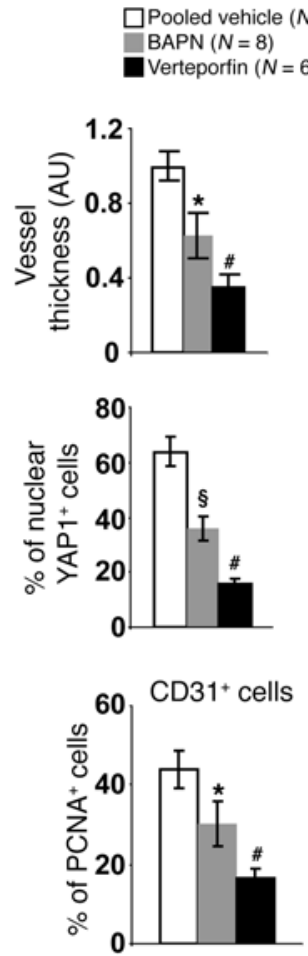
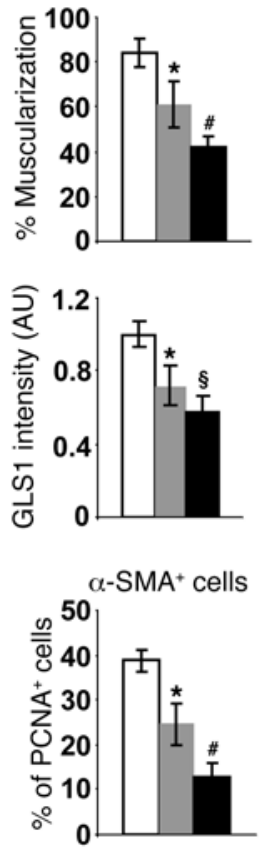

B

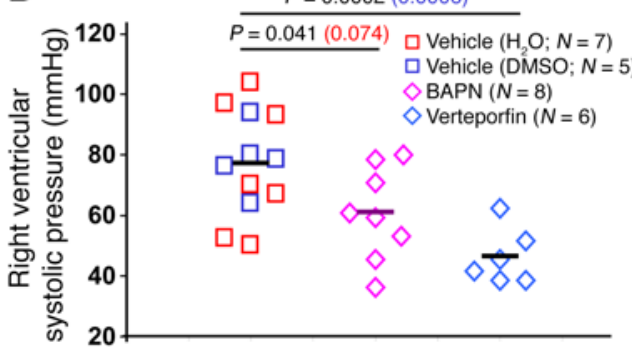

C Right ventricular hypertrophy

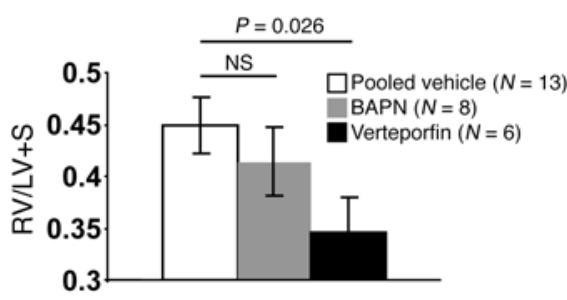

Figure 12. Manipulation of vascular mechanotransduction in vivo controls pulmonary vascular proliferation and PH. Following monocrotaline exposure, rats were treated with daily BAPN $(n=8)$ versus vehicle $(\mathbf{A} ; n=7)$ or with daily i.p. injections of separate verteporfin $(n=6)$ versus separate vehicle $(\mathbf{B} ; n=6)$. (A) H\&E stain and coimmunofluorescence microscopy revealed a decrease of vessel thickness and muscularization as well as a decrease of YAP1+ cells, GLS1 vascular intensity, and CD31/PCNA and $\alpha$-SMA/PCNA double-positive cells in BAPN- and verteporfin-treated rats compared with vehicle controls. (B and C) Verteporfin reduced PAH severity, as quantified by RVSP (B) and right ventricular hypertrophy (Fulton index, RV/LV+S) (C). Similar trends were observed with BAPN treatment. In all panels, mean expression in control groups was assigned a fold change of 1 , to which relevant samples were compared. $P$ values were derived from 1-way ANOVA and post-hoc Tukey's tests $\left({ }^{*} P<0.05,{ }^{\$} P<0.01,{ }^{\#} P<0.001\right)$. Scale bar: $50 \mu \mathrm{m}$. See also Supplemental Figure 12.

molecular pathogenesis of HIV-PAH. By establishing the actions of YAP/TAZ and GLS1 in primate and human models of PAH secondary to HIV or SIV infection, our findings provide long-awaited evidence that, beyond histopathologic associations, the molecular and cellular pathophenotypes active in this subtype of PAH overlap with other PAH forms and may be amenable to treatment with similarly targeted therapeutics. These results portend future fundamental discoveries - specifically in elucidating how HIV infection directly or indirectly activates vessel stiffening, YAP/ $\mathrm{TAZ}$, and consequent metabolic derangement in the pulmonary vasculature. Because of the lack of reliable rodent models of HIV infection, our study also demonstrates the feasibility of discerning molecular mechanism from PAH modeling in primates in general.
Finally, the identification of the mechanoactivation of glutaminolysis in PH sets the stage to develop novel clinical management strategies in PH. Our results (Figures 11-14) demonstrate that a range of functionally connected targets related to matrix remodeling and glutaminolysis may also show promise for further therapeutic development in $\mathrm{PH}$. The improvement of hemodynamic and histologic indices of $\mathrm{PH}$ in monocrotaline-exposed rats with BAPN (Figures 11 and 12) reinforces the importance of collagen cross-linking and ECM remodeling in PH pathogenesis and is consistent with prior studies inhibiting Lox in chronic hypoxic $\mathrm{PH}(1,3$, 47). However, therapeutic use of a specific Lox inhibitor alone may suffer from modest efficacy (i.e., right ventricular remodeling; Figure 12), potentially due to the importance of several other Lox fam- 
A

C968 i.p. injections (10 mg/kg/d) or vehicle i.p. injections

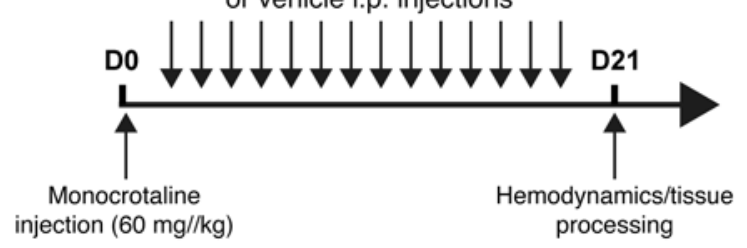

B

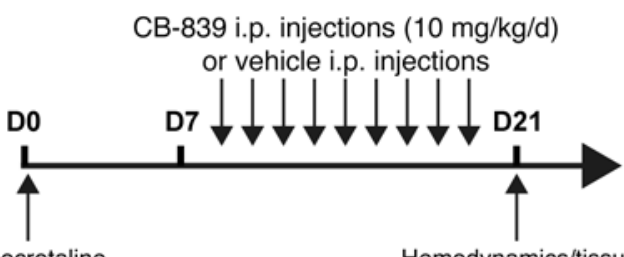

Monocrotaline injection $(60 \mathrm{mg} / \mathrm{kg})$
Hemodynamics/tissue processing

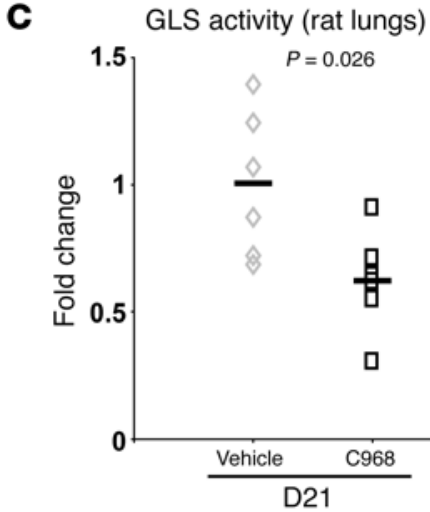

E
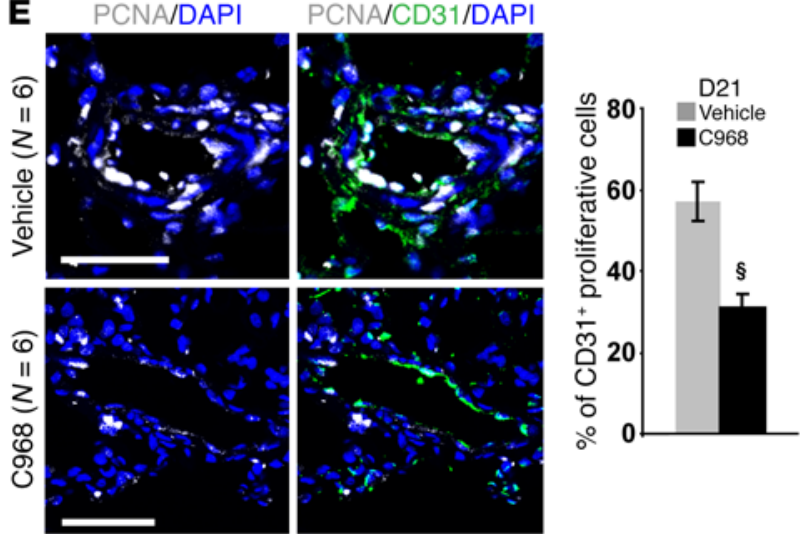

G
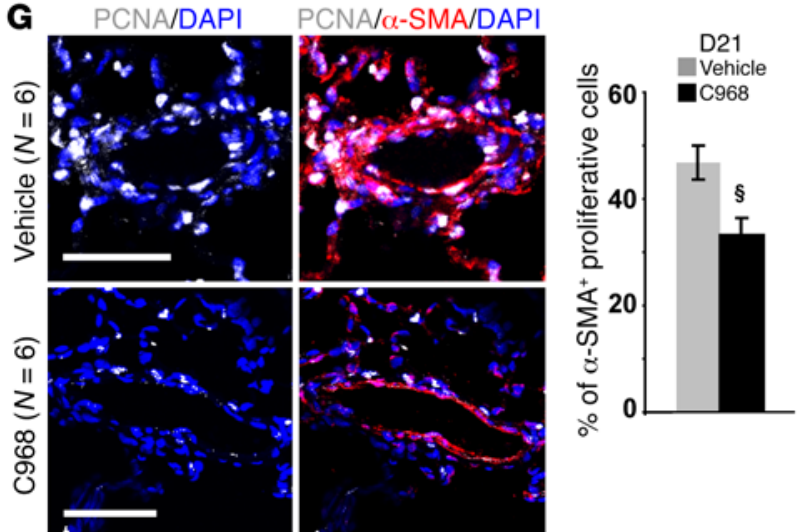

D GLS activity (rat lungs)

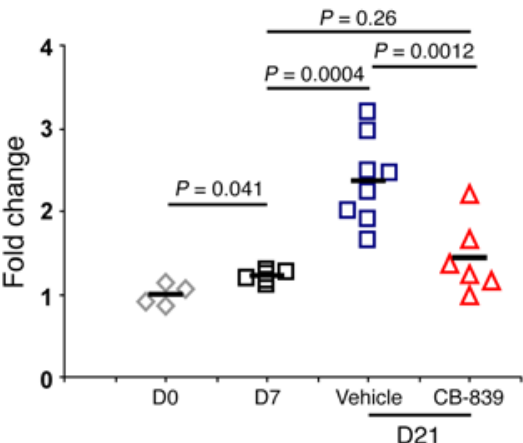

\section{F $\quad v W F / K i 67 / D A P I$}
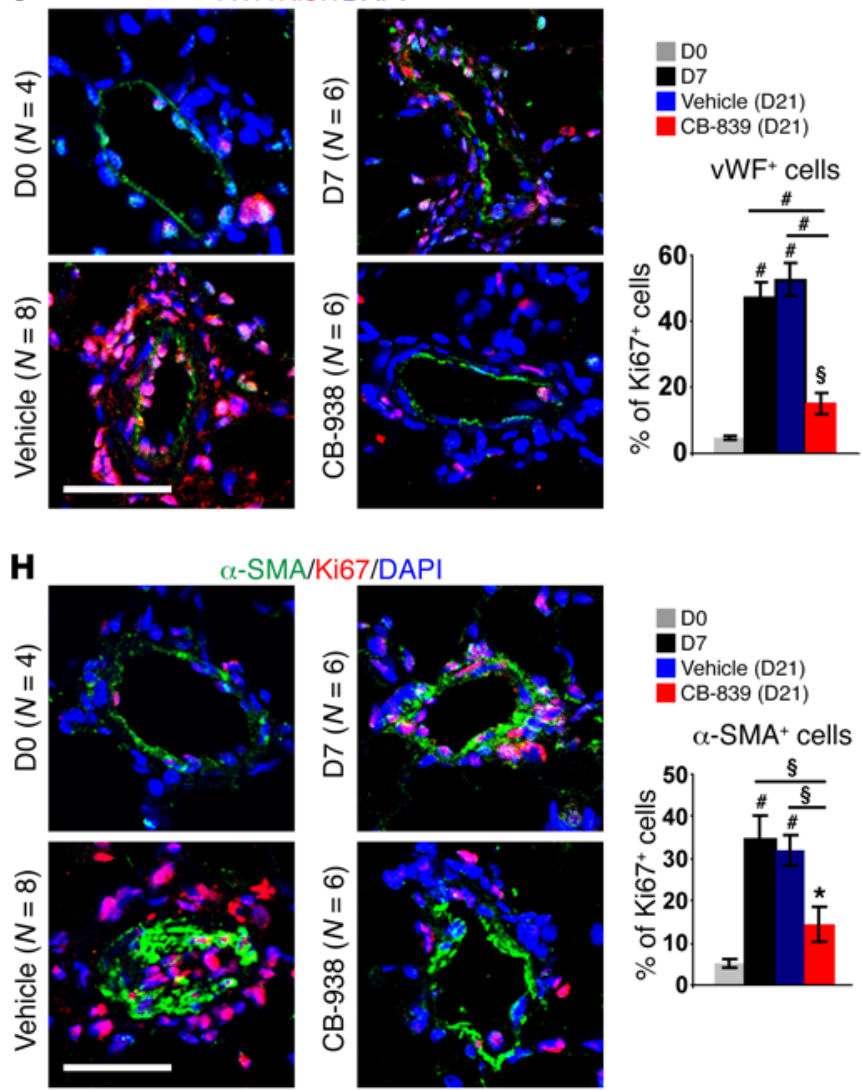

Figure 13. Pharmacologic inhibition of GLS1 in monocrotaline-exposed rats decreases glutaminolysis and consequent pulmonary vascular cell proliferation. (A and B) After monocrotaline administration, rats were treated with daily i.p. injections of 2 pharmacologic inhibitors of GLS1. Vehicle ( $n=6)$ versus C968 $(n=6)(\mathbf{A})$ and vehicle $(n=8)$ versus CB-839 ( $n=6)$ (B) were compared using either a disease prevention (C968) or disease reversal (CB-839) dosing protocol. (C and D) Either C968 (C) or CB-839 (D) decreased GLS activity in lungs of monocrotaline-exposed rats. (E-H) Coimmunofluorescence microscopy revealed Ki-67/PCNA-positive proliferating cells in diseased pulmonary arterioles (vehicle). Either C968 or CB-839 reduced the number of Ki-67/PCNApositive cells in $\alpha-S M A^{+}$medial $(\mathbf{G}$ and $\mathbf{H})$ and $C D 31^{+}$or $v W F^{+}$endothelial ( $\mathbf{E}$ and $\mathbf{F}$ ) compartments. In all panels, mean expression in control groups was assigned a fold change of 1 , to which relevant samples were compared. Data are expressed as the mean $\pm \mathrm{SEM}\left({ }^{*} P<0.05,{ }^{\S} P<0.01,{ }^{\#} P<0.001\right)$. Paired samples were compared by a 2-tailed Student's $t$ test, while 1-way ANOVA and post-hoc Tukey's tests were used for group comparisons. Scale bars: $50 \mu \mathrm{m}$. 
A

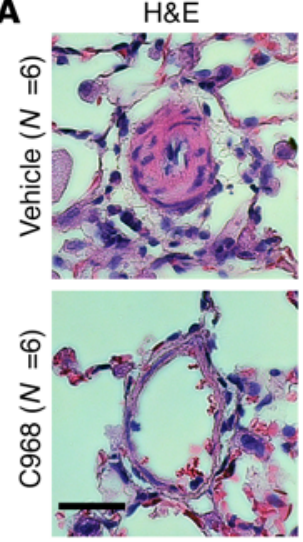

C

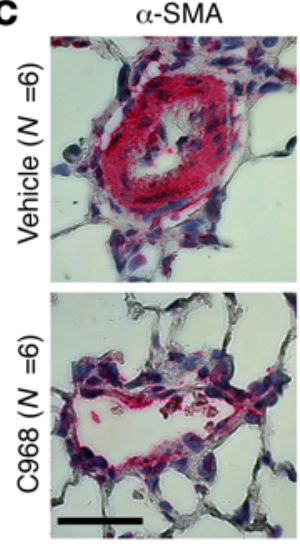

E

Right ventricular systolic pressure

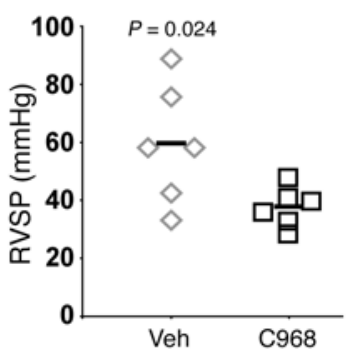

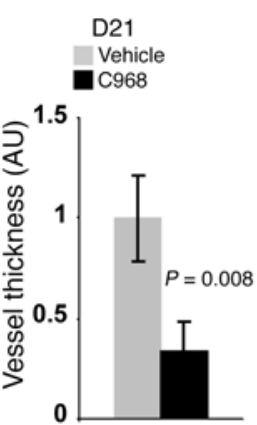
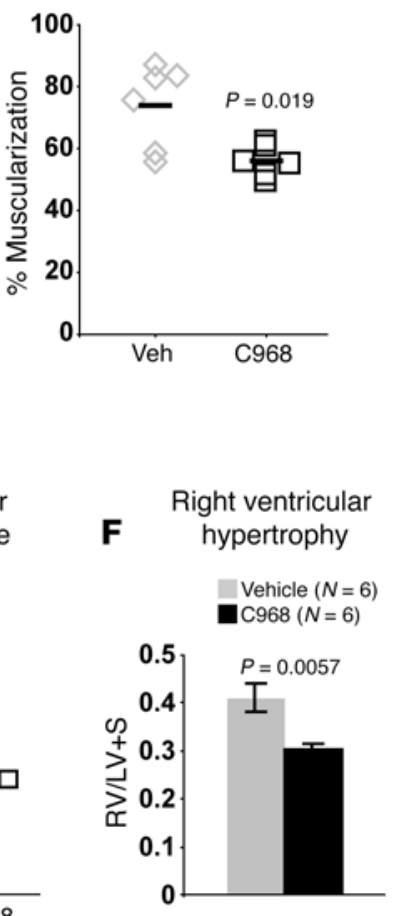

B
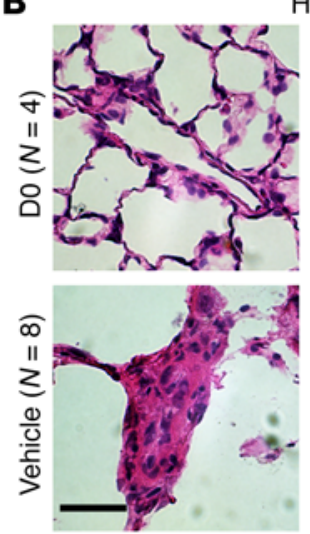

$H \& E$
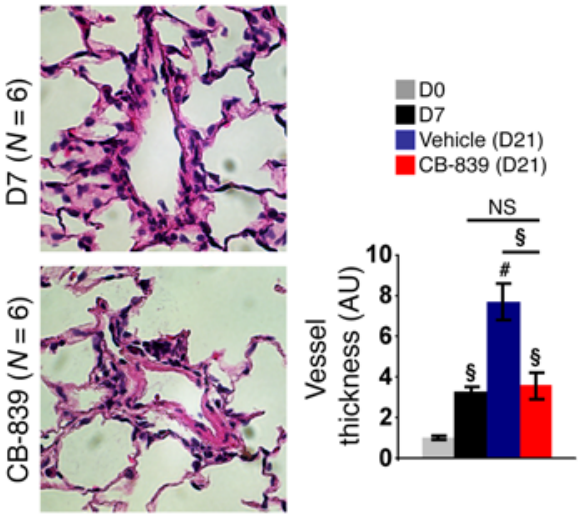

D

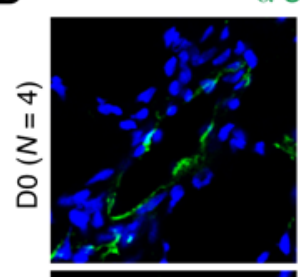

$\alpha$-SMA/DAPI
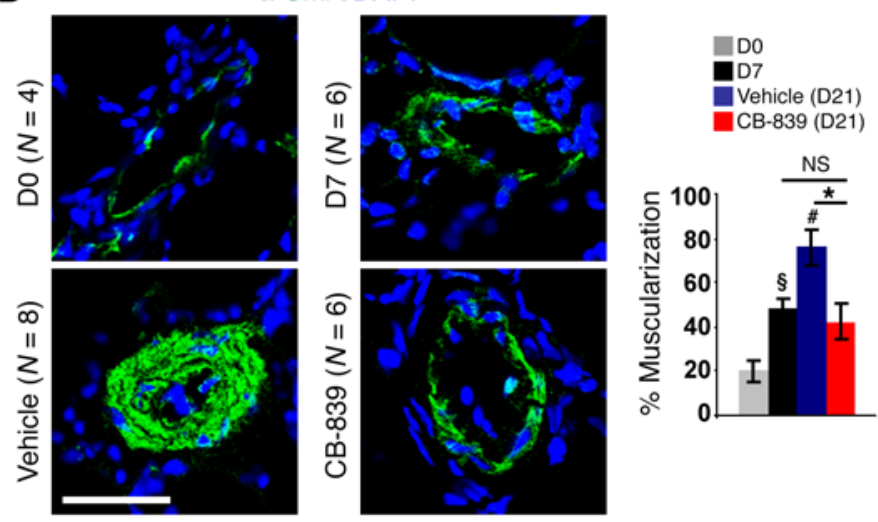

Right ventricular systolic pressure

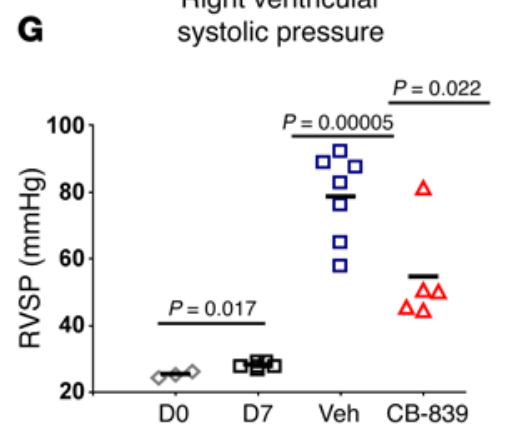

Figure 14. Pharmacologic inhibition of GLS1 in monocrotaline-exposed rats ameliorates PH in vivo. Following monocrotaline injection, via either a disease prevention (C968) or disease reversal (CB-839) dosing protocol (cohort sizes defined in each panel), inhibition of glutaminolysis ameliorated PH severity, as quantified by vascular remodeling ( $\mathbf{A}$ and $\mathbf{B}$ ), arteriolar muscularization (C and D), RVSP (E and $\mathbf{G})$, and right ventricular hypertrophy (Fulton index, RV/LV+S) (F and $\mathbf{H})$. In all panels, mean expression in control groups was assigned a fold change of 1 , to which relevant samples were compared. Data are expressed as the mean \pm SEM $\left({ }^{*} P<0.05,{ }^{\circledR} P<0.01\right.$, $\left.{ }^{\#} P<0.001\right)$. Paired samples were compared by a 2-tailed Student's $t$ test, while 1 -way ANOVA and post-hoc Tukey's tests were used for group comparisons. Scale bars: $50 \mu \mathrm{m}$. See also Supplemental Figures 13 and 14.

ily members that may show complementary function (3). Yet, when coupled with targeting of the downstream YAP/TAZ-GLS1 axis, an additive or perhaps synergistic therapeutic benefit may emerge in inhibiting pulmonary vascular proliferation and remodeling. This may be particularly evident with YAP, given the emerging beneficial effects even beyond metabolism of altering Hippo signaling in the pulmonary vasculature $(1,48)$ and the robust improvement of severe rodent PH using the YAP inhibitor verteporfin alone (Figures 11 and 12). Importantly, verteporfin is already approved by the US FDA as an intravenous medication for the treatment of age-related macular degeneration (49). Moreover, an oral form of the GLS1 inhibitor CB-839 is undergoing active clinical development in early human clinical trials for cancer therapy (Clinical Trial NCT02071862). Therefore, a repurposing of verteporfin and CB-839 for treatment of $\mathrm{PH}$, either separately or together with possible optimization for tissue-specific delivery, presents a rare opportunity to offer novel therapeutics in this disease without the delay of decades to develop small-molecular inhibitors de novo. 
Pulmonary vascular stiffness controls glutaminolysis and anaplerosis in $\mathrm{PH}$

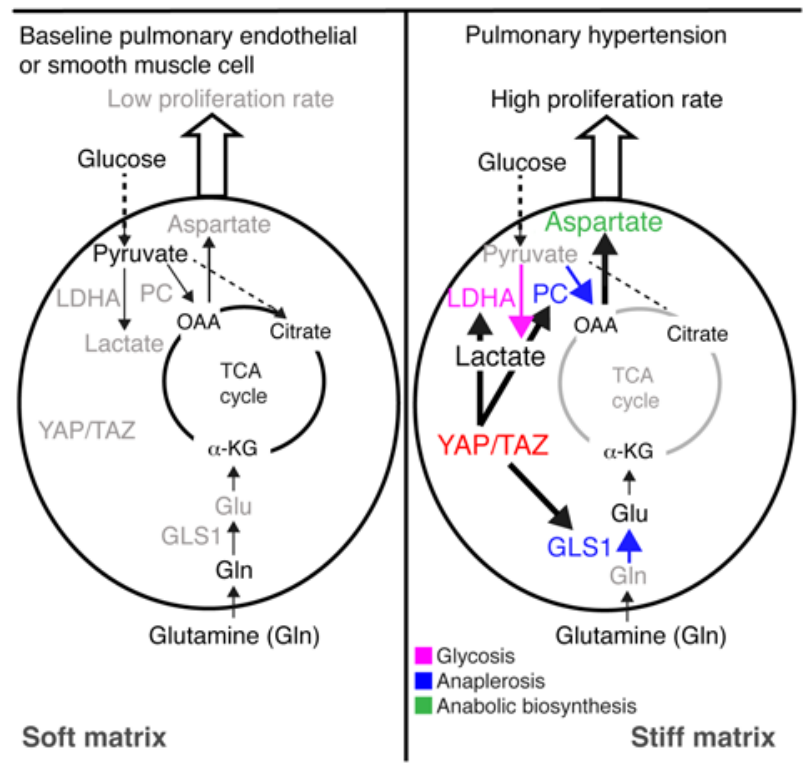

In sum, our findings define the YAP/TAZ-dependent activation of glutaminolysis and anaplerosis as a central pathogenic mechanism by which vessel stiffness can stimulate cellular proliferation in PH. These results carry broad implications for our fundamental understanding of how cells balance their energetic demands with extracellular mechanical cues. These findings endorse the rapid application of novel pharmacologic inhibitors to target the metabolic effects of vascular stiffness and thus prevent or reverse human $\mathrm{PH}$. Moreover, such translational studies in PH may have broad application to other human metabolic conditions in which such mechanosensitive interactions may serve as crucial origins of disease.

\section{Methods}

Supplemental Methods are available online with this article. See complete uncut gels in the supplemental material.

Cell culture reagents for studies dependent on matrix stiffness. At baseline, cultured cells were grown in collagen-coated plastic (50 $\mu \mathrm{g}$ / $\mathrm{ml}$ ) at $37^{\circ} \mathrm{C}$ in a humidified $5 \% \mathrm{CO}_{2}$ atmosphere. Collagen-coated hydrogel preplated in culture wells (Matrigen) was generated from a mix of acrylamide and bis-acrylamide coated with collagen. Cells were cultured, passaged, and harvested while on top of the hydrogel, using standard cell culture techniques as we previously described (1). According to the manufacturer's descriptions, the Young's (elastic) modulus of a hydrogel was determined based on a published protocol (50), with a modification of assuming a Poisson ratio of 0.48 for the acrylamide mixture. Specifically, the hydrogels were fully equilibrated in PBS at room temperature ( $>24$ hours). A sphere (glass or carbide steel) of less than $1 \mathrm{~mm}$ diameter was placed on the surface of the hydrogel, and the depth of indentation was measured. To determine the Young's (elastic) modulus using the measurement of this depth, Hertz's theory was applied with an adjustment for buoyancy.

Targeted LC-MS/MS. Metabolite extraction was performed essentially as described with minor modifications (51). Briefly, metabolites were extracted from cultured cells and $\mathrm{CD} 31^{+}$cells on dry ice using $80 \%$ aqueous methanol precooled at $-80^{\circ} \mathrm{C}$. Metabolites were
Figure 15. Model of pathogenic mechanoactivation of YAP/TAZ and key metabolic enzymes by pulmonary vascular stiffness in order to control glutaminolysis and PH. In nondiseased and compliant pulmonary arterioles, endothelial and smooth muscle cells undergo a basal level of proliferation (left diagram). Pulmonary vascular ECM stiffening mechanoactivates YAP/TAZ to induce key metabolic enzymes GLS1, PC, and LDHA and to promote glutaminolysis and glycolysis. Such glutaminolysis sustains the metabolic needs of hyperproliferative vascular cells, thus driving overt pulmonary vascular remodeling and $\mathrm{PH}$ (right diagram). extracted from plasma precleared by centrifugation at 20,000 $g$ for 10 minutes at $4^{\circ} \mathrm{C}$. Supernatants were extracted with 4 volumes of $100 \%$ methanol precooled at $-80^{\circ} \mathrm{C}$ for 4 hours at $-80^{\circ} \mathrm{C}$. An internal standard, $\left[{ }^{13} \mathrm{C}_{4}\right]$-2-oxoglutarate $\left(\left[{ }^{13} \mathrm{C}_{4}\right]-2 \mathrm{OG}\right)$ (Cambridge Isotope Laboratories), was added during metabolite extraction. Insoluble material from both cell and plasma extractions was removed by centrifugation at 20,000 $\mathrm{g}$ for 15 minutes at $4^{\circ} \mathrm{C}$. The supernatant was analyzed by targeted LC-MS/MS as previously described (52). Metabolites were separated using a ZIC-HILIC stationary phase $(150 \mathrm{~mm} \times$ $2.1 \mathrm{~mm} \times 3.5 \mathrm{~mm}$; Merck). The MS parameters were optimized using a glutamine standard solution. Monitored mass transitions were 87 to 87 (pyruvate), 115 to $73+99$ (succinate), 132 to 88 (aspartate), 145 to 101 (2OG), 145 to 127 (glutamine), 146 to 128 (glutamate), and 149 to $105\left(\left[{ }^{13} \mathrm{C}_{4}\right]-2 \mathrm{OG}\right)$. Mass transitions and retention time windows were confirmed by the analysis of neat and matrix-spiked standards. Peak areas were quantified by Xcalibur Software (Thermo Fisher Scientific) and manually reviewed.

Extracellular flux analyses. PAECs (30,000 cells per well) or PASMCs (50,000 cells per well) were plated in Seahorse Bioscience 24-well plates precoated with $20 \mu \mathrm{l}$ of soft or stiff hydrogel (as described above). After overnight incubation to allow attachment, cells were washed 2 times in assay medium (DMEM without phenol red or pyruvate containing $0.5 \%$ dialyzed FBS and $0.1 \mathrm{mg} / \mathrm{ml}$ uridine at $\mathrm{pH}$ 7.4; Seahorse Biosciences) and incubated in $500 \mu \mathrm{l}$ of fresh assay medium. Oxygen consumption rate and extracellular acidification rate (a surrogate marker of glycolysis) were measured on an XF24 or XFe24 Analyzer (Seahorse Biosciences). Mitochondrial and glycolytic stress assays were performed according to the manufacturer's protocols. Oxygen consumption rate and extracellular acidification rate were normalized to cell count measured after assay completion.

Inhibition of Lox or YAP in $\mathrm{PH}$ rats. To induce $\mathrm{PH}$, male SpragueDawley rats (10-14 weeks old) were injected i.p. with $60 \mathrm{mg} / \mathrm{kg}$ monocrotaline (Sigma-Aldrich). After 1 day, rats underwent i.p. injection daily with $25 \mathrm{mg} / \mathrm{kg}$ of verteporfin (Active Biochemicals Co. Ltd.) versus vehicle control. In parallel but in separate rat cohorts, 1 day after monocrotaline injection, $\beta$-aminopropionitrile (BAPN; 
$100 \mathrm{mg} / \mathrm{kg} / \mathrm{d}$; Sigma-Aldrich) was administered in drinking water versus drinking water alone. In all cohorts, on day 21 after monocrotaline injection (1 day after the last administration of BAPN, verteporfin, or vehicle controls), right heart catheterization was performed in all cohorts followed by harvesting of lung tissue and CD $31^{+}$cells for RNA or protein extraction, paraffin embedding, or cryopreservation with OCT (Sigma-Aldrich), as we described (1).

Inhibition of GLS1 in $\mathrm{PH}$ rats. To induce $\mathrm{PH}$, male SpragueDawley rats (10-14 weeks old) were injected i.p. with $60 \mathrm{mg} / \mathrm{kg}$ monocrotaline (Sigma-Aldrich). After 2 days, serial i.p. injections were given daily of C968 (10 mg/kg; Sigma-Aldrich) versus vehicle control, and 7 days after monocrotaline injection, serial i.p. injections were given daily of CB-839 (10 mg/kg; Selleck Chemicals) versus vehicle control. In all cohorts, on day 21 after monocrotaline injection, right heart catheterization was performed followed by harvesting of lung tissue and $\mathrm{CD} 31^{+}$cells for RNA or protein extraction, paraffin embedding, or cryopreservation with OCT (SigmaAldrich), as we described (1).

Human subjects. Informed consent was obtained for all study procedures. For formalin-fixed paraffin-embedded lung samples, human PH specimens were collected from unused or discarded surgical samples (Supplemental Table 2), some of which we have described previously (53); nondiseased human lung specimens from the New England Organ Bank have been described (53). For plasma harvesting and analysis described in Figure 8, E-G, individuals were chosen with clinically significant dyspnea and undergoing right heart catheterization at the Brigham and Women's Hospital, Boston, Massachusetts, USA (Supplemental Table 3; some of whom were described in ref. 31). Subjects were stratified by the presence or absence of clinical $\mathrm{PH}$, as defined by elevated mean pulmonary arterial pressure $(\mathrm{mPAP}) \geq 25 \mathrm{mmHg}$. For measurements of pulmonary artery compliance, a cohort of $42 \mathrm{HIV}$-infected individuals underwent pulmonary arterial catheterization at the University of Pittsburgh. A diagnosis of PAH ( $\mathrm{mPAP} \geq 25 \mathrm{mmHg}$ ) was made in 11 individuals (Supplemental Table 4). Based on these invasive hemodynamic measurements, pulmonary arterial compliance was calculated by stroke volume/pulse pressure, as described previously (54). Finally, for human subjects in whom peripheral plasma was analyzed for circulating metabolites (Figure 10 and Supplemental Table 5), a separate cohort of HIV-infected individuals were recruited at UCSF either with documented PAH (assessed by invasive pulmonary arterial catheterization, $\mathrm{mPAP} \geq 25 \mathrm{mmHg}$ ) or without $\mathrm{PAH}$ (assessed either with invasive pulmonary arterial catheterization, $\operatorname{mPAP}<25$ $\mathrm{mmHg}$, or by noninvasive echocardiographic estimation of pulmonary arterial systolic pressure $<40 \mathrm{mmHg}$ ).

Human plasma sampling. To collect blood from subjects from the main pulmonary artery, clinically indicated right heart catheterization procedures were performed by standard protocol via a right internal jugular approach under fluoroscopic guidance, as we described (53). The catheter was positioned into the main pulmonary artery, as confirmed by fluoroscopy and hemodynamic waveforms. Blood was drawn from the distal port and collected in vacutainer tubes with $\mathrm{K}^{+}$-EDTA. Plasma was extracted after centrifugation of blood, followed by storage at $-80^{\circ} \mathrm{C}$. A similar approach was used to collect venous peripheral plasma from HIV-positive subjects, as we described (55).

Statistics. Cell culture experiments were performed at least 3 times and at least in triplicate for each replicate. The number of ani- mals in each group was calculated to measure at least a $20 \%$ difference between the means of experimental and control groups with a power of $80 \%$ and SD of $10 \%$. The number of unique patient samples for this study was determined primarily by clinical availability. In situ expression/histologic analyses of both rodent and human tissue, and pulmonary vascular hemodynamics in mice and rats, were performed in a blinded fashion. Numerical quantifications for in vitro experiments using cultured cells or in situ quantifications of transcript/miR expression represent mean \pm SD. Numerical quantifications for physiologic experiments using rodents or human reagents represent mean \pm SEM. Immunoblot images are representative of experiments that were repeated at least 3 times. Micrographs are representative of experiments in each relevant cohort. Normality of data distribution was determined by Shapiro-Wilk testing. Paired samples were compared by a 2-tailed Student's $t$ test for normally distributed data, while Mann-Whitney $U$ nonparametric testing was used for non-normally distributed data. For comparisons among groups, 1-way ANOVA and post hoc Tukey testing was performed. A $P$ value less than 0.05 was considered significant.

Study approval. All animal experiments were approved by the Harvard Center for Comparative Medicine and the University of Pittsburgh. All experimental procedures involving the use of human tissue and plasma and the study of invasive and noninvasive hemodynamics included the relevant receipt of written informed consent and were approved by institutional review boards at Partners Healthcare, Boston Children's Hospital, UCSF, UCLA, and the University of Pittsburgh, as well as the New England Organ Bank. Ethical approval for this study and informed consent conformed to the standards of the Declaration of Helsinki.

Accession codes. Expression array data were deposited in the NCBI Gene Expression Omnibus (http://www.ncbi.nlm.nih.gov/geo/) under series GSE75793.

\section{Author contributions}

TB, WMO, KAC, and SYC conceived and designed the experiments. KAC performed the in silico analyses. TB, WMO, JF, QY, JZ, YT, YT, YYZ, CG, MAS, JL, and KAN provided the experimental infrastructure and performed the experiments. MAS, SR, RRV, AM, VNP, PYH, TDM, KAN, RS, RS, WDW, DJR, SOV, KJH, and ABW obtained human and animal $\mathrm{PH}$ samples. SP performed atomic force microscopy analysis. MS, ZQ, and JG performed rodent echocardiography. TB, WMO, KAC, JF, and SYC wrote the manuscript. All authors participated in interpreting the results and revising the manuscript.

\section{Acknowledgments}

We thank L. Fredenburgh for technical advice and M. Jain and J. Snow for critical manuscript advice. The authors acknowledge the IRCAN's Molecular and Cellular Core Imaging (PICMI) Facility (supported by le Cancéropole PACA, la Région PACA, le Conseil Départementale 06, l'INSERM, ARC, IBiSA, and the Conseil Départemental 06 de la Région PACA). This work was supported by NIH grants HL096834 and HL124021 (to S.Y. Chan); the American Heart Association (Post-doctoral Award), the Ligue Nationale contre le Cancer, and Fondation Bettencourt-Schueller (to T. Bertero); the French National Research Agency (ANR-11LABX-0028-01) and Association pour la Recherche sur le Can- 
cer (ARC) grant PJA20131200325 (to C. Gaggioli and T. Bertero); NIH grants P01-HL103455 (to A. Morris and M.A. Simon), R56-HL126525 (to K.A. Norris), and R01-HL090339 (to A. Morris); grant from Gilead Sciences, Inc. (to A. Morris); NIH grants HL61795, HL48743, HL108630, and GM107618 (to J. Loscalzo); NIH grant HL007633 and HL128802 (to W.M. Oldham); and NIH grant HL121174 (to J. Fessel).
Address correspondence to: Stephen Y. Chan, Center for Pulmonary Vascular Biology and Medicine, Pittsburgh Heart, Lung, Blood, and Vascular Medicine Institute, Division of Cardiology, Department of Medicine, University of Pittsburgh School of Medicine, University of Pittsburgh Medical Center, 200 Lothrop Street BST1704.2, Pittsburgh, Pennsylvania 15261, USA. Phone: 412.383.6990; E-mail: chansy@pitt.edu.
1. Bertero T, et al. Matrix remodeling promotes pulmonary hypertension through feedback mechanoactivation of the YAP/TAZ-miR-130/301 circuit. Cell Rep. 2015;13(5):1016-1032.

2. Lammers S, Scott D, Hunter K, Tan W, Shandas $\mathrm{R}$, Stenmark KR. Mechanics and function of the pulmonary vasculature: implications for pulmonary vascular disease and right ventricular function. Compr Physiol. 2012;2(1):295-319.

3. Nave AH, et al. Lysyl oxidases play a causal role in vascular remodeling in clinical and experimental pulmonary arterial hypertension. Arterioscler Thromb Vasc Biol. 2014;34(7):1446-1458.

4. Dupont S, et al. Role of YAP/TAZ in mechanotransduction. Nature. 2011;474(7350):179-183.

5. Cottrill KA, Chan SY. Metabolic dysfunction in pulmonary hypertension: the expanding relevance of the Warburg effect. Eur J Clin Invest. 2013;43(8):855-865.

6. Paulin R, Michelakis ED. The metabolic theory of pulmonary arterial hypertension. Circ Res. 2014;115(1):148-164.

7. Zhao L, et al. The zinc transporter ZIP12 regulates the pulmonary vascular response to chronic hypoxia. Nature. 2015;524(7565):356-360.

8. Diebold I, et al. BMPR2 preserves mitochondrial function and DNA during reoxygenation to promote endothelial cell survival and reverse pulmonary hypertension. Cell Metab. 2015;21(4):596-608.

9. Wang W, et al. AMPK modulates Hippo pathway activity to regulate energy homeostasis. Nat Cell Biol. 2015;17(4):490-499.

10. Mo JS, et al. Cellular energy stress induces AMPK-mediated regulation of YAP and the Hippo pathway. Nat Cell Biol. 2015;17(4):500-510.

11. Le A, et al. Glucose-independent glutamine metabolism via TCA cycling for proliferation and survival in B cells. Cell Metab. 2012;15(1):110-121.

12. Lunt SY, Vander Heiden MG. Aerobic glycolysis: meeting the metabolic requirements of cell proliferation. Annu Rev Cell Dev Biol. 2011;27:441-464.

13. Zhao Y, Butler EB, Tan M. Targeting cellular metabolism to improve cancer therapeutics. Cell Death Dis. 2013;4:e532.

14. Sullivan LB, Gui DY, Hosios AM, Bush LN, Freinkman E, Vander Heiden MG. Supporting aspartate biosynthesis is an essential function of respiration in proliferating cells. Cell. 2015;162(3):552-563.

15. Birsoy K, Wang T, Chen WW, Freinkman E, Abu-Remaileh M, Sabatini DM. An essential role of the mitochondrial electron transport chain in cell proliferation is to enable aspartate synthesis. Cell. 2015;162(3):540-551.

16. Piao L, Fang YH, Parikh K, Ryan JJ, Toth PT, Archer SL. Cardiac glutaminolysis: a maladap- tive cancer metabolism pathway in the right ventricle in pulmonary hypertension. J Mol Med. 2013;91(10):1185-1197.

17. Robinson MM, et al. Novel mechanism of inhibition of rat kidney-type glutaminase by bis-2-(5-phenylacetamido-1,2,4-thiadiazol-2-yl)ethyl sulfide (BPTES). Biochem J. 2007;406(3):407-414.

18. Ahluwalia GS, Grem JL, Hao Z, Cooney DA. Metabolism and action of amino acid analog anti-cancer agents. Pharmacol Ther. 1990;46(2):243-271.

19. Wang JB, et al. Targeting mitochondrial glutaminase activity inhibits oncogenic transformation. Cancer Cell. 2010;18(3):207-219.

20. Croft D, et al. The Reactome pathway knowledgebase. Nucleic Acids Res. 2014;42(Database issue):D472-D477.

21. Piccolo S, Dupont S, Cordenonsi M. The biology of YAP/TAZ: hippo signaling and beyond. Physiol Rev. 2014;94(4):1287-1312.

22. Morrell NW, et al. Cellular and molecular basis of pulmonary arterial hypertension. J Am Coll Cardiol. 2009;54(1 suppl):S20-S31.

23. George MP, et al. Physiologic changes in a nonhuman primate model of HIV-associated pulmonary arterial hypertension. Am J Respir Cell Mol Biol. 2013;48(3):374-381.

24. Barnett CF, Hsue PY. Human immunodeficiency virus-associated pulmonary arterial hypertension. Clin Chest Med. 2013;34(2):283-292.

25. Iwatsuki K, Cardinale GJ, Spector S, Udenfriend S. Reduction of blood pressure and vascular collagen in hypertensive rats by beta-aminopropionitrile. Proc Natl Acad Sci U S A. 1977;74(1):360-362.

26. Felley-Bosco E, Stahel R. Hippo/YAP pathway for targeted therapy. Transl Lung Cancer Res. 2014;3(2):75-83.

27. Liang N, et al. Regulation of YAP by mTOR and autophagy reveals a therapeutic target of tuberous sclerosis complex. JExp Med. 2014;211(11):2249-2263.

28. Gross MI, et al. Antitumor activity of the glutaminase inhibitor CB-839 in triple-negative breast cancer. Mol Cancer Ther. 2014;13(4):890-901.

29. Tung JC, et al. Tumor mechanics and metabolic dysfunction. Free Radic Biol Med. 2015;79:269-280

30. Archer SL, Fang YH, Ryan JJ, Piao L. Metabolism and bioenergetics in the right ventricle and pulmonary vasculature in pulmonary hypertension. Pulm Circ. 2013;3(1):144-152.

31. White K, et al. Genetic and hypoxic alterations of the microRNA-210-ISCU1/2 axis promote iron-sulfur deficiency and pulmonary hypertension. EMBO Mol Med. 2015;7(6):695-713.

32. Pickup MW, Mouw JK, Weaver VM. The extracellular matrix modulates the hallmarks of cancer.
EMBO Rep. 2014;15(12):1243-1253.

33. Sakao S, Taraseviciene-Stewart L, Lee JD, Wood K, Cool CD, Voelkel NF. Initial apoptosis is followed by increased proliferation of apoptosis-resistant endothelial cells. FASEB J. 2005;19(9):1178-1180.

34. Michelakis ED. Spatio-temporal diversity of apoptosis within the vascular wall in pulmonary arterial hypertension: heterogeneous BMP signaling may have therapeutic implications. Circ Res. 2006;98(2):172-175.

35. Long L, et al. Selective enhancement of endothelial BMPR-II with BMP9 reverses pulmonary arterial hypertension. Nat Med. 2015;21(7):777-785.

36. Ranchoux B, et al. Endothelial-to-mesenchymal transition in pulmonary hypertension. Circulation. 2015;131(11):1006-1018.

37. Hopper RK, et al. In pulmonary arterial hypertension, reduced BMPR2 promotes endothelialto-mesenchymal transition via HMGA1 and its target slug. Circulation. 2016;133(18):1783-1794.

38. Kim J, et al. Restoration of impaired endothelial myocyte enhancer factor 2 function rescues pulmonary arterial hypertension. Circulation. 2015;131(2):190-199.

39. Xu W, Erzurum SC. Endothelial cell energy metabolism, proliferation, and apoptosis in pulmonary hypertension. Compr Physiol. 2011;1(1):357-372.

40. Enzo E, et al. Aerobic glycolysis tunes YAP/ TAZ transcriptional activity. EMBO J. 2015;34(10):1349-1370.

41. Sorrentino G, et al. Metabolic control of YAP and TAZ by the mevalonate pathway. Nat Cell Biol. 2014;16(4):357-366.

42. Su T, Bondar T, Zhou X, Zhang C, He H, Medzhitov R. Two-signal requirement for growthpromoting function of Yap in hepatocytes. Elife. 2015;4:e2948.

43. Fitamant J, et al. YAP inhibition restores hepatocyte differentiation in advanced HCC, leading to tumor regression. Cell Rep. 2015;10(10):1692-1707.

44. Fessel JP, et al. Metabolomic analysis of bone morphogenetic protein receptor type 2 mutations in human pulmonary endothelium reveals widespread metabolic reprogramming. Pulm Circ. 2012;2(2):201-213.

45. van den Heuvel AP, Jing J, Wooster RF, Bachman KE. Analysis of glutamine dependency in nonsmall cell lung cancer: GLS1 splice variant GAC is essential for cancer cell growth. Cancer Biol Ther. 2012;13(12):1185-1194.

46. Archer SL, Gomberg-Maitland M, Maitland ML, Rich S, Garcia JG, Weir EK. Mitochondrial metabolism, redox signaling, and fusion: a mitochondria-ROS-HIF-1 $\alpha$-Kv1.5 O2-sensing pathway at the intersection of pulmonary hypertension 
and cancer. Am J Physiol Heart Circ Physiol. 2008;294(2):H570-H578.

47. Kerr JS, Riley DJ, Frank MM, Trelstad RL, Frankel HM. Reduction of chronic hypoxic pulmonary hypertension in the rat by $\beta$-aminopropionitrile. J Appl Physiol Respir Environ Exerc Physiol. 1984;57(6):1760-1766.

48. Kudryashova TV, et al. HIPPO-integrin linked kinase crosstalk controls self-sustaining proliferation survival in pulmonary hypertension [published online ahead of print April 27, 2016]. Am J Respir Crit Care Med. doi:10.1164/ rccm.201510-2003OC.

49. Kent DL. Age-related macular degenera- tion: beyond anti-angiogenesis. Mol Vis. 2014;20:46-55.

50. Damljanović V, Lagerholm BC, Jacobson K. Bulk and micropatterned conjugation of extracellular matrix proteins to characterized polyacrylamide substrates for cell mechanotransduction assays. BioTechniques. 2005;39(6):847-851.

51. Yuan M, Breitkopf SB, Yang X, Asara JM. A positive/negative ion-switching, targeted mass spectrometry-based metabolomics platform for bodily fluids, cells, and fresh and fixed tissue. Nat Protoc. 2012;7(5):872-881.

52. Oldham WM, Clish CB, Yang Y, Loscalzo J. Hypoxia-mediated increases in L-2-hydroxygluta- rate coordinate the metabolic response to reductive stress. Cell Metab. 2015;22(2):291-303.

53. Bertero T, et al. Systems-level regulation of microRNA networks by miR-130/301 promotes pulmonary hypertension. J Clin Invest. 2014;124(8):3514-3528.

54. Pellegrini P, et al. Prognostic relevance of pulmonary arterial compliance in patients with chronic heart failure. Chest. 2014;145(5):1064-1070.

55. Parikh VN, et al. Brief report: coordinated modulation of circulating miR-21 in HIV, HIV-associated pulmonary arterial hypertension, and HIV/ Hepatitis $\mathrm{C}$ virus coinfection. J Acquir Immune Defic Syndr. 2015;70(3):236-241. 